\title{
Descriptions and a Key to Cultivars of J apanese Cedar Cultivated in the Eastern United States
}

Robert J. Rouse, ${ }^{1}$ Paul R. Fantz, and Ted E. Bilderback

AdDitional INDEX WORDS. conifer, Cryptomeria, gymnosperm, ornamental, morphology, taxonomy, Taxodiaceae, C upressaceae

Summary. Japanese cedar, Cryptomeria japonica (Thunb. ex L.f.) D. D on [C upressaceae Bartling, formerly assigned to T axodiaceae Warm.] is increasing in popularity as a landscape plant in the eastern U nited States. A taxonomic study of cultivars grown in the eastern U nited States was conducted. Forty-five cultivars were recognized. E ach cultivar bears synonymy, a quantitative morphological description newly described from field data, herbarium vouchers, references to original literature and observational notes. A glossary of taxonomic terms relevant to Cryptomeria is presented. A taxonomic key is presented for segregation of cultivars that should assist professional plantsmen in identification of taxa cultivated in the eastern $U$ nited States.

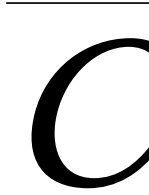

ryptomeria japonica (japanese cedar or cryptomeria) is indigenous to J apan. D irr (1990) noted that japanese cedar is underused in the southern U nited States and has potential for use in any landscape situation. Cultivars of $C$. japonica performed well in heavy red clay soils during both prolonged dry and wet periods, exhibited remarkable tolerance to hot, humid, summer conditions, and have been regarded as nearly pest free (Tripp, 1993). Japanese cedar is considered as one of the better gymnosperms adapted for the eastern U nited States, and is increasing in popularity as a landscape plant (Tripp, 1993).

Tripp (1993) reported that several new cultivars introduced into the U nited States lacked descriptions. Rouse et al. (1997) established that japanese cedar in the eastern U nited States included a large number of named cultivars that needed an organized inventory of taxa, correct scientific names and synonyms, quantitative descriptions, taxonomic keys for segregation of taxa, and documentation of existing germplasm. The objective of this paper is to provide an inventory of cultivars of japanese cedar in cultivation in the eastern U nited States, original quantitative morphological descriptions of each cultivar, and a taxonomic key segregating the cultivars. This would provide a useful resource in assisting professional plantsmen in segregation and identification of cultivars of japanese cedar.

Department of H orticultural Science, Box 7609, N orth Carolina State U niversity, Raleigh, NC 27695-7609.

Research Project N C 06104 funded in part by the N orth C arolinaA gricultural Research Service, Raleigh, N C 27695-7643. U se of nursery and trade names in this publication does not imply endorsement by the N CARS of the nurseries or the products mentioned nor criticism of similar ones not mentioned. The cost of publishing this paper was defrayed in part by the payment of page charges. Under postal regulations, this paper therefore must be hereby marked adverti sement solely to indicate this fact.

${ }^{1} \mathrm{~N}$ ational Arborist Association (Blithewold M ansion and Gardens). Current address: 9 J ohn Street, N arragansett, RI 02882. 
Table 1. M orphological characteristics examined for C ryptomeria.

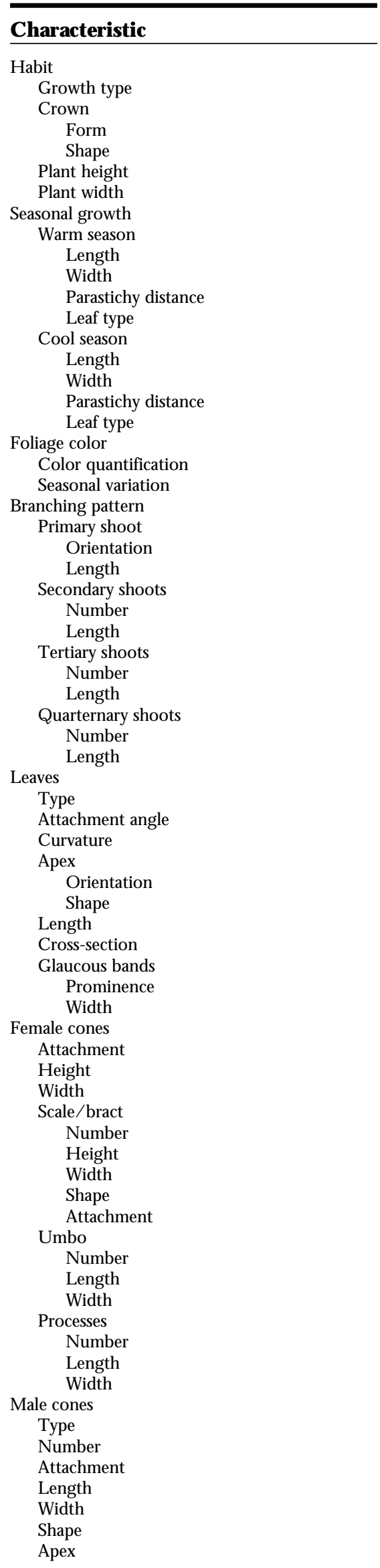

Materials and methods

$D$ atawerecollected by Rousefrom named cultivars of mature plants of japanese cedar at multiplesitesin 199496. Limited availability of many cultivars restricted data collected to three to six plants per cultivar. H owever, many cultivars of japanese cedar are rarein the landscape/ nurseryindustries. Therefore, sampling was limited to only one or two plants. All plants sampled were labeled with cultivar names and were at least 4 years old.

Standardized datasheetswere prepared listing variousmorphological characters appropriate for plants in thisfamily (Table 1). M orphological terminology used followed standard taxonomic references (Eiselt, 1960; $\mathrm{H}$ arris and H arris, 1994; Vidakovic, 1991). Vegetative shoot data were recorded from 12 to 15 different locations per plant. $D$ ata on branch growth patterns were recorded from six to eight different primary branches and leaf data were recorded from 10 to 20 leaves per location. Strobili data were recorded from 12 to 15 strobili per plant when available, yet many cultivars lacked male and/ or female strobili. Foliage color was quantified utilizing the $M$ unsell color chart (Wilde and Voigt, 1977). $D$ ata sampling replicated on different plants of cultivar in different locations, when available, up to six replications. $D$ ata replication after three plants rarely produced new results in data obtained.

Q uantitativedescriptionswereprepared for each cultivar from the data collected and compared to prepare a taxonomic key of segregation. Herbarium vouchers (Rouse numbers) of each plant were obtained and will be deposited at the herbarium of $\mathrm{N}$ orth Carolina State U niversity and the $\mathrm{Na}$ tional Arboretum.

\section{Results and discussion}

Professional plantsmen working with japanese cedar are interested in the proper identification of cultivars. Plantsmen wanting to view a number of different cultivars can visit those sites examined in this study that maintain large collections of cultivars of japanese cedar. These included the Atlanta Botanical Garden (Atlanta, Ga.), M orris Arboretum (Philadelphia, Pa.), the JC Raulston Arboretum of $\mathrm{N}$ orth Carolina State U niversity (Raleigh, N.C.), and the U.S. N ational Arboretum (Washington, D.C.).
This study recognized 45 distinct cultivars. All cultivar descriptions are based upon firsthand field datafrom live plants. Cultivar descriptions herein are expanded and more quantitative than descriptions found in standard gymnosperm references ( $D$ en Ouden and Boom, 1978; H ornibrook, 1938; Krüssmann, 1985; Tripp, 1993; Welch, 1979, 1991).

A nalysis of shoots of japanese cedar required modification of some of the standardized morphological terminology used for C ryptomeria. A glossary of modified terminology is included in Table 2. Terminology for growth forms and crowns has been modified. Shoots of cultivars of Cryptomeria exhibited distinct seasonal growth periods that could be characterized, but which have not been to date ( $D$ en O uden and Boom, 1978; H ornibrook, 1938; Krüssmann, 1985; Tripp, 1993; Welch, 1979). Thus, seasonal growth terminology is newly defined (T able 2 ).

Foliage color variation in the species occurs as a result of age differences, seasonal differences, and cultural differences. Cultivar variation occurs mostly in hues of green. Age differentiation is typically from lighter green (new foliage) to darker green (mature foliage). N ewly emerged foliage is creamy-white or silvery-white in some cultivars. Winter color is influenced by exposure to winter sun, low temperatures, wind protection, and severity of winter conditions. Winter foliage color exhibits bronze to brown to purplish-red coloration, but these colors denoted for cultivars may not be consistent. Foliage color is defined using the M unsell system (Wilde and Voigt, 1977). The notation for any chromatic color is hue value/ chroma( $\mathrm{HV} / \mathrm{C})$, wherehuerepresents one of the basic primary colors (red, green, yellow, blue, purple), value the degree of lightness $(<5)$ or darkness $(>5)$, and chroma the strength of saturation (increases with higher number). H owever, color chips are lacking for white pigmentations, thus our terms creamy-white and silvery-white are defined along with $M$ unsell notations for other colors in Table 1.

Literaturedescriptionsavailablefor cultivars describe adult leaves as awns, needles, awl-shaped, acicular or subulate (Den Ouden and Boom, 1978; Krüssmann, 1985; Tripp, 1993; Welch, 1991, 1993). Different authors, describing the same cultivar, will often use Text continued on p. 265 
T able 2. Terminology used in describing the morphology of japanese cedar. M easurements cited are in metric units (25.4 $\mathrm{mm}=1.0 \mathrm{inch} ; 0.3 \mathrm{~m}=\mathbf{1 . 0} \mathrm{ft}$ ) and standard taxonomic format ( $\mathrm{x}-\mathrm{y}$ is common measurement range). $\mathrm{Crown} \mathrm{H} / \mathrm{W}=$ the height to width ratio. Foliage color defined follows Wilde and Voigt (1977) notation of hue value/chroma (e.g., 5G 6/4-6 is color chart $5 \mathrm{G}$ reen with a degree of lightness val ue of 6 and a chroma or saturation strength of 4 or $\mathbf{6}$ ). C olor chips are lacking for whitish pigmentations and are defined here.

\section{Characteristic and definition}

Growth forms

Compact shrub: 1-3 m tall $\times 1-3 \mathrm{~m}$ wide, accompanied by increased growth rate, with either linear or saber leaves.

D warf shrub: $1 \mathrm{~m}$ tall $\times 1 \mathrm{~m}$ wide, with slow growth rate and saber leaves.

Tree: Pyramidal to conical, to $50 \mathrm{~m}$ tall $\times 3-5 \mathrm{~m}$ wide, with saber leaves.

Crowns

Columnar: Shrub with narrow width, 0.5-4 $\mathrm{m}$ tall (H/ W 8:1), acute apex and cuneate base.

Conical: Cone-shaped tree, to $30 \mathrm{~m}$ tall $\times 3 \mathrm{~m}$ wide $(\mathrm{H} / \mathrm{W}$ 10:1); broader basally than columnar, but narrower than pyramidal.

Cylindrical: Shrub with width to $1 \mathrm{~m}$ wide throughout height (H/ W 4:1), apex obtuse and base narrow.

0 void: Shrub widest at middle to below, to $3 \mathrm{~m}$ tall $\times 2 \mathrm{~m}$ wide $(\mathrm{H} / \mathrm{W}$ 1.5:1), apex tapering obtuse.

Globose: Round shrub, to $2 \mathrm{~m}$ tall $\times 3 \mathrm{~m}$ wide $(\mathrm{H} / \mathrm{W} 0.6: 1)$, midsection broad and flattened.

M ound: Pincushion shrub with broad base, to $0.3 \mathrm{~m}$ tall $\times 0.5 \mathrm{~m}$ wide (H/ W 0.6:1), apex obtusely rounded.

$O$ val: O val shrub to $3 \mathrm{~m}$ tall $\times 2 \mathrm{~m}$ wide (H/ W 1.5:1), midsection broad and rounded.

Pyramidal: Tree with triangular shape, to $30 \mathrm{~m}$ tall $\times 6 \mathrm{~m}$ wide (H/ W 5:1); broader basally than conical.

Subglobose: Flattened globular shrub, to $2 \mathrm{~m}$ tall $\times 2 \mathrm{~m}$ wide (H / W 1:1); apex narrower than globular and broader basally.

Branches

Cool season growth: Growth with short internode length, small leaves, narrow shoot width and short shoot length.

Primary shoot: Central axis of a spray composed of several seasonal growth cycles; longest shoot with attached secondary shoots.

Q uarternary shoot: First order of shoot borne from tertiary shoot axis, exhibiting only one seasonal growth cycle.

Seasonal growth: Growth occurring during one season, divided into a cool and a warm season growth phase.

Secondary shoot: First order of shoots borne from the primary shoot.

Shoot width: Distance between two tangential lines parallel to branch axis, touching the most exterior leaves.

Spray: M ain branch axis and associated secondary/ tertiary shoots; terminal portion of branch that is entirely green and extending beyond the lignified portion of the branch.

Tertiary shoot: First order of shoot borne from the secondary shoot axis.

Warm season growth: Growth with long internode length, large leaves, broad shoot width and long shoot length.

Foliage color

Bluish-green: 5G 6/ 4-6

Bronze: 2.5YR 4/ 4-8 and 3/ 4-6

Brown: 7.5YR 5/ 4-8 and 4/ 4

C reamy-white: White color with slight yellow pigmentation becoming light green with maturation.

Dark green: $7.5 \mathrm{GY}$ 4/ 4-6

Greenish-yellow: $2.5 \mathrm{GY} 7 / 6-10$

Light green: $5 \mathrm{GY} 5 / 6-8$

M edium green: $5 \mathrm{GY} 4 / 6-8$

Purplish-red: 10R 4/ 6-10

Silvery-white: Whitish color lacking any green pigmentation with maturation.

Yellow: 5 Y 8/ 8-10

Leaves

Appressed: Attachment angle of $5-20^{\circ}$, growing with at least half of leaf touching the twig axis.

Ascending: Attachment angle of $20-85^{\circ}$, causing leaf apex to point forward and only the basal portion touching the twig axis.

Incurved: Leaf shaft forms a concave arc toward the twig axis.

Linear: Leaves are flat, straight to recurved, 4-25 mm long, 2-4 mm wide, acute to acuminate, straight to inflexed, attached at angle of 45-95.

Perpendicular: Attachment angle $85-95^{\circ}$, causing leaves to appear at a right angle with twig axis.

Recurved: Leaf shaft forms a slight convex arc, bending backwards from twig axis.

Saber: L eaves swordlike, quadrangular or dorsiventrally compressed, inflexed, 7-24 mm long, 1.5-3 mm wide, acute to acuminate, reflexed or inflexed (acicular, needle of some authors).

Shaft: Free portion of leaf blade not attached to twig.

Short saber: Leaves swordlike, keeled, straight to slightly incurved, 1-6 mm long, 1-4 mm wide, acute, straight or inflexed (awn or subulate of some authors). Spiral: Leaf shaft twisted counterclockwise or clockwise around the twig axis, twisting varying from $0.25-1$ complete rotation.

Straight saber: Leaf shaft lacks an arc, and forms a straight line from base to apex.

Cones or strobili

Conical: $M$ ale strobilus acute with width less than half the length.

O blong: M ale strobilus obtuse with length 2-4 times longer than width.

$O$ void: $M$ ale strobilus obtuse, broadly elliptic, with width over half the length

Processes: Acuminate apical protrusions from the scale/ bracts of female cones, 1-6 mm long, to $1.5 \mathrm{~mm}$ wide.

Scale/ bracts: Combination of an ovulate scale and a bract fused together at the base to midsection, leathery becoming woody at maturation, upon which the seeds occur basally; commonly 4-8 $\mathrm{mm}$ long, 4-8 $\mathrm{mm}$ wide.

Spikelike: Terminal cluster of male cones formed when nearly each axis of a dwarf shoot initiates a male strobilus.

Strobili complex: Globose cluster of male strobili with bases appearing fused, formed when a strobili-bearing shoot fails to elongate.

U mbo: Scale/ bract apex, straight to reflexed, acute. 
Fig. 1. An alphabetized list of selected cultivar names of Cryptomeria japonica. Measurements cited are in metric units $(25.4 \mathrm{~mm}=1.0 \mathrm{inch} ; 0.3$ $\mathrm{m}=1.0 \mathrm{ft}$ ) and standard taxonomic format (number in parenthesis is measurement seldom encountered; $x-y$ is common measurement range). Voucher numbers cited are R ouse collections that will be deposited at the herbarium of $\mathrm{N}$ orth $\mathrm{C}$ arolina State $\mathrm{U}$ niversity and the $\mathrm{N}$ ational Arboretum. C ultivar names recognized include synonymy [alternative names applied in $\mathrm{H}$ orticulture (H ort.) or in literature] enclosed in brackets, a quantitative morphological description, herbarium vouchers, authorities and observations. Cultivar names recognized as synonyms include the authority, and the correct cultivar name following the equal symbol. Article 17.9 of the International C ode of N omenclature (IC N CP; T rehane, 1995) requires cultivar names published after $1 \mathrm{~J}$ an. 1959 to be words in a modern language, not Latin; new names are proposed in these cases known.

\section{'Araucarioides' [Cryptomeria araucarioi des Siebold; 'Enko-sugi'; 'Yenko-sugi']. Tree 11-15 m tall, 2-3 m wide, narrow pyramidal. Primary shoots ascending, (10) 25-56 cm long; second- ary shoots ascending, 0-10 (14), 7-35 cm long; tertiary shoots $0-1$ (6), (2) $5-11 \mathrm{~cm}$ long; quaternary shoots lacking; seasonal growth conspicuous; warm season growth 3.5-9.5 cm long, 8-16 mm wide; cool season growth $0.5-6 \mathrm{~cm}$ long, 3-7 mm wide. Foliage color brown in winter, dark green in summer, medium green on new growth. Warm season leaves saber, slightly incurved, 7-14 mm long, apex inflexed, acute to acuminate, attachment ascending 30-45\% angle. Cool season leaves short saber, straight to deeply incurved, 2-5 $\mathrm{mm}$ long, apex straight, acute, attachment appressed to ascending 5-45\% angle. Female strobili rare on young plants. $M$ ale strobili rare on young plants. Vouchers: $178,210,267$. N amed as species (Siebold, 1856); as cultivar (D allimore and J ackson, 1948). \\ O bservations: Tree recognized easily by snakelike branches, but confused with 'D acrydioides' which has shorter leaves and tufted secondary branches.}

'Aurea' [var. aurea Beissner; 'O gonsugi']. Tree to $20 \mathrm{~m}$ tall, $4 \mathrm{~m}$ wide, broad conical to narrow pyramidal, central leader strong. Primary shoot $10-15 \mathrm{~cm}$ long; secondary shoots 7-20, 4-11.5 cm long; tertiary shoots $0-16,0.6-15 \mathrm{~cm}$ long; quaternary shoots $0-1$, to $0.6 \mathrm{~cm}$ long; seasonal growth conspicuous; warm season growth 1.5-4.5 cm long, 12-17 $\mathrm{mm}$ wide; cool season growth $1-1.5 \mathrm{~cm}$ long, 5-7 mm wide. Foliage color bronze in winter, light green in summer, yellow on new growth, darkening with first warm night temperatures. Warm season leaves straight saber, 7-20 mm long, apex straight, acuminate, attachment ascending $30-45 \%$ angle. Cool season leaves short saber, straight, attachment 3-4 mm long, apex straight, acute, ascending 30-45\% angle. Female strobili not observed. $M$ ale strobili not observed. Vouchers: 232, 272. $\mathrm{N}$ amed as variety (Beissner, 1891); as cultivar (D en Ouden and Boom, 1965).

O bservations: 'Aurea' and 'Sekkan' are shrubs distinguished easily by the yellow variegated new growth. H owever, 'Aurea' quickly becomes a light green by summer, whereas 'Sekkan' retains a yellowish-green color throughout summer.

'Bandai-sugi' [ var. bandai sugi; 'Itosugi']. Compact shrub to small tree, very slow growing to $1 \mathrm{~m}$ tall, $1.5 \mathrm{~m}$ wide, irregular. Primary shoot 2-14 cm long; secondary shoots $0.4-6 \mathrm{~cm}$ long, congested along axis, only warm season 20-80, only cool season (0) 10-20; tertiary shoots $0-12,0.3-2.5 \mathrm{~cm}$ long, congested along axis; quaternary shoots lacking; seasonal growth difference conspicuous; warm season growth 1-1.5 $\mathrm{cm}$ long, 11-19 mm wide; cool season growth 0.5-1.5 cm long, 4-7 mm wide. Foliage color purplish-red in winter, medium green in summer, light green on new growth. Warm season leaves saber, straight to slightly recurved, 11-19 mm long, apex straight, acuminate, attachment ascending $60-80 \%$ angle. Cool season leaves short saber, straight, 1-3 $\mathrm{mm}$ long, apex straight, acute, attachment ascending 45-80\% angle. Female strobili rare. M ale strobili rare. Voucher: 219. $\mathrm{N}$ amed as variety (H ornibrook, 1938); as cultivar (D en Ouden and Boom, 1965).

O bservations: 'Bandai-sugi' and

'Yokohama' are slow growing dwarf shrubs of those cultivars with irregular crowns and profuse cool season secondary shoots. 'Bandai-sugi' is distinguished by the longer leaves, large number of secondary shoots, and mature size to nearly a meter tall.

'Benjamin Franklin' H atch (1985) ['Bennie's Best']. Tree to $20 \mathrm{~m}$ tall, 3-4 $\mathrm{m}$ wide, narrowly conical, central leader straight, dead and tertiary shoots not persisting. Primary shoot ascending, 13$29 \mathrm{~cm}$ long; secondary shoots 7-16, pendulous, $0.5-15.4 \mathrm{~cm}$ long; tertiary shoots $0-16$, pendulous, $0.4-9 \mathrm{~cm}$ long; quaternary shoots lacking; seasonal growth conspicuous; warm season growth
3.5-6 cm long, 8-12 mm wide; cool season growth $0.5-7 \mathrm{~cm}$ long, 2-6 mm wide. Foliage color dark green in winter, medium green in summer, light green on new growth. Warm season leaves saber, straight to deeply incurved, 9-17 mm long, apex straight or inflexed, acute, attachment ascending 30-45\% angle. Cool season leaves short saber, straight to slightly incurved, 2-5 mm long, apex straight, acute to acuminate, attachment ascending $45 \%$ angle. Female strobili persistent on upper crown of mature trees. $M$ ale strobili persistent on upper crown of mature trees. Voucher: 181.

O bservations: A conical tree distinguished by the dark green winter color, lack of quarternary shoots, and shedding of inner dead branches.

'Bennie's Best' H ort. = 'B enjamin
Franklin'

'Birodo-sugi' H ort. = 'C ompressa'

'Black D ragon' Tripp (1993). Compact shrub to $2 \mathrm{~m}$ tall, $0.5 \mathrm{~m}$ wide, columnar. Primary shoot 7-16 cm long; secondary shoots 2-22, 0.5-4 cm long, congested along axis; tertiary shoots $0-6,0.4-3.2$ $\mathrm{cm}$; quaternary shoots lacking; seasonal growth difference conspicuous; warm season growth $0.8-3.2 \mathrm{~cm}$ long, 9-25 $\mathrm{mm}$ wide; cool season growth $0.7-1.3 \mathrm{~cm}$ long, 3-6 mm wide. Foliage color dark green in winter and summer, bluish-green on new growth. Warm season leaves saber, straight to slightly incurved, 8-18 $\mathrm{mm}$ long, apex straight to inflexed, acute to acuminate, attachment ascending 30$45 \%$ angle. Cool season leaves short saber, straight, 1-3 mm long, apex straight, acute to acuminate, attachment 30-45\% angle. Female strobili rare. $\mathrm{M}$ ale strobili persistent, terminal spikelike clusters on secondary and tertiary shoots, 1-11 per cluster, ovoid, 4-5 mm long, 2-3 mm wide, apex obtuse. Vouchers: 198, 227.

O bservations: Cool season growth very short. Similar to 'Giokumo' but distinguished by narrower columnar form and dark green foliage.

'Bloomers Witches Broom' H ort.

['N ational Arboretum Witches Broom'; 'Rein's D ense J ade']. Compact shrub to $1.5 \mathrm{~m}$ tall, $1 \mathrm{~m}$ wide, subglobose to broad pyramidal. Primary shoot ascending, 8.5$19.5 \mathrm{~cm}$ long; secondary shoots 7-23, $0.3-18 \mathrm{~cm}$ long; tertiary shoots $0-15$, $0.3-8 \mathrm{~cm}$ long; quaternary shoots $0-7$, 0.3-4 cm long; seasonal growth conspicuous; warm season growth $1.5-9 \mathrm{~cm}$ long, 7-11 mm wide; cool season growth 0.9$2.9 \mathrm{~cm}$ long, 3-4 mm wide. Foliage color 
medium green in winter and summer, bluish-green on new growth. Warm season leaves saber, slightly incurved, 8$11 \mathrm{~mm}$ long, apex straight to inflexed, acute, attachment ascending 30-45\% angle. Cool season leaves short saber, slightly incurved, 2-4 mm long, apex inflexed, acute, attachment appressed 5$20 \%$ angle. Female strobili rare. M ale strobili rare. Vouchers: 193, 203, 247, 280.

O bservations: D istinguished by appressed cool season leaves (or narrow cool seasonal growth). 'L ittle Diamond' appears to be a diminutive form of this cultivar.

'B uckiscope' H ort. Compact shrub, adult, $1.5 \mathrm{~m}$ tall, $0.75 \mathrm{~m}$ wide, narrowly conical. Primary shoot $7.5-13 \mathrm{~cm}$ long; secondary shoots $8-14,1-6 \mathrm{~cm}$ long; tertiary shoots $0-5,0.7-2.3 \mathrm{~cm}$ long; quaternary shoots rare, $0-1,0.4-0.7 \mathrm{~cm}$ long; seasonal growth conspicuous; warm season growth 1.5-7.2 cm long, 9-14 $\mathrm{mm}$ wide; cool season growth $1.0-1.5 \mathrm{~cm}$ long, 3-4 mm wide. Foliage color bronze in winter, medium green in summer, light green on new growth. Warm season leaves saber, slightly incurved, 6-9 mm long, apex straight, acuminate, attachment ascending $45 \%$ angle. Cool season leaves short saber, slightly incurved, 1-3 mm long, apex straight, acute, attachment appressed to ascending at 5-30\% angle. Female strobili rare. M ale strobili rare. Voucher: 240.

O bservations: $M$ ore plants required for additional data for adequate characterization. Known from Atlanta Botanical Garden.

'Compressa' D en O uden (1949). ['Birodo-sugi']. D warf shrub to $1 \mathrm{~m}$ tall, $0.5 \mathrm{~m}$ wide, ovoid. Primary shoot ascending, 1.4-5 (6.5) cm long; secondary shoots $1-10,0.4-5 \mathrm{~cm}$ long; tertiary shoots $0-3,0.3-0.6 \mathrm{~cm}$ long, not exserted beyond margin of shrub; quaternary shoots lacking; seasonal growth conspicuous; warm season growth $0.8-3.5 \mathrm{~cm}$ long, $15-27 \mathrm{~mm}$ wide; cool season growth $0.7-1.8 \mathrm{~cm}$ long, 5-7 mm wide. Foliage color bronze in winter, dark green in summer, bluish-green on new growth. Warm season leaves linear, straight to slightly incurved, 7-13 mm long, apex straight, acute to acuminate, attachment ascending to perpendicular, 60-90\% angle. Cool season leaves short saber, straight to slightly recurved, $1.5-6 \mathrm{~mm}$ long, apex reflexed, acute to acuminate, attachment ascending to perpendicular, 45-90\% Female strobili rare. $M$ ale strobili rare. Vouchers: 202, 208, 243, 263.
O bservations: 'C ompressa' is distinguished by warm season linear leaves and cool season short saber leaves. Often confused with 'Vilmoriniana' which is a broader and taller shrub with nodding terminal shoots exserted beyond the rest of the foliage and short saber warm season leaves.

'C ristata' Beissner (1901). ['Sekkasugi'; 'Sekkwia-sugi']. Small tree to $6 \mathrm{~m}$ tall, $2.5 \mathrm{~m}$ wide, pyramidal, trunk knotted or twisted irregularly. Primary shoots ascending, $5-22 \mathrm{~cm}$ long; secondary shoots 3-30, 4-16 cm long, occasionally fasciated; tertiary shoots 0 18, 1-6.5 cm long, occasionally fasciated; quaternary shoots lacking; seasonal growth not conspicuous; nonfasciated growth 5-10 cm long, 15-30 mm wide; fasciated shoots $6-15.5 \mathrm{~cm}$ long, 2.5$10.5 \mathrm{~cm}$ wide. Foliage color brown in winter, medium green in summer, light green on new growth. N onfasciated leaves saber, straight to slightly incurved, 7-18 mm long, apex straight to inflexed, acute to acuminate, attachment ascending 45-60\% angle. Fasciated leaves saber, straight to slightly incurved, 4-18 mm long, apex straight to inflexed, acute to acuminate, appressed $45 \%$ angle. Female strobili rare. $M$ ale strobili rare. Vouchers: 284, 288, 292.

O bservations: Distinguished by the larger fasciated (cockscomb-like) shoots than 'Kilmacurragh'. The twisted or knotted trunks of 'C ristata' and 'Kilmacurragh' appear to be the result of grafting during development.

\section{'D acrydioides' [ C ryptomeria}

dacrydi oi desC arr.]. Tree, adult, 11-15 m tall, 2-3 $\mathrm{m}$ wide, narrow pyramidal. Primary shoots ascending, (10) 25-56 $\mathrm{cm}$ long; secondary shoots ascending, 15-27, 1.4-6 cm long; tertiary shoots 0 1 (3), $0.5-5 \mathrm{~cm}$ long; quaternary shoots lacking; warm seasonal growth $1-6 \mathrm{~cm}$ long, 7-11 mm wide; seasonal growth conspicuous; cool seasonal growth 0.7-3 $\mathrm{cm}$ long, 3-6 mm wide. Foliage color purplish-red in winter, dark green in summer, medium green on new growth. Warm season leaves saber, slightly incurved, 5-7 mm long, apex inflexed, acuminate, attachment ascending 45$60 \%$ angle. $\mathrm{C}$ ool season leaves saber to short saber, slightly incurved, 2-6 mm long, apex inflexed, acute, attachment appressed to ascending 5-45\% angle. Female strobili rare on young plants. $\mathrm{M}$ ale strobili rare on young plants. Voucher: 230. N amed as species (C arrière, 1867); as cultivar (D allimore and J ackson, 1948).

O bservations: Tree with snakelike branches bearing tufted secondary branches, and with shorter leaves than 'Araucarioides'.

'D warf Whitetip' = proposed name for 'N ana Albospica' in accordance with Article 17.9 of the ICN CP.

'E legans' [C ryptomeria elegans; 'H imesugi']. Small tree (juvenile $3 \mathrm{~m}$ tall, $1 \mathrm{~m}$ wide), columnar to narrowly conical. Primary shoots perpendicular, $19-27 \mathrm{~cm}$ long; secondary shoots 10-19, 1.7-12 $\mathrm{cm}$ long; tertiary shoots $0-9,1.5-7 \mathrm{~cm}$ long; quaternary shoots lacking; seasonal growth conspicuous; warm season growth 1.5-4.9 cm long, 13-52 mm wide; cool season growth $0.5-0.7 \mathrm{~cm}$ long, 4-6 $\mathrm{mm}$ wide. Foliage color bronze to purplish-red in winter, medium green in summer, medium green on new growth. Warm season leaves linear, straight to slightly recurved, 8-25 mm long, apex inflexed, acute to acuminate, attachment perpendicular, 80-95\% angle. Cool season leaves linear, straight to recurved, 1-5 mm long, apex straight, acute to acuminate, attachment ascending to perpendicular, $60-90 \%$ angle. Female strobili rare. $\mathrm{M}$ ale strobili persistent, clustered on primary shoots, proximal, 1-8 per cluster; conical, 3-7 $\mathrm{mm}$ long, 2-3 mm wide, apex acute. Vouchers: 186, 237. $\mathrm{N}$ amed as species ( $M$ akoy, 1864); as cultivar (D allimore and Jackson, 1948).

O bservations: Small tree with narrow crown and leaves linear. Distinguished from 'Elegans Viridis' by winter foliage becoming bronze to purplish-red.

'E legans A urea' Welch (1966) = 'Golden Elegance'. Compact shrub to small tree (juvenile, $3 \mathrm{~m}$ tall, $1 \mathrm{~m}$ wide), columnar to narrowly conical. Primary shoot perpendicular, $11.5-46 \mathrm{~cm}$ long; secondary shoots $8-40,0.6-16 \mathrm{~cm}$ long; tertiary shoots $0-12.0 .6-4.2 \mathrm{~cm}$ long, distal ends pendulous; quaternary shoots lacking; seasonal growth conspicuous; warm season growth $1.4-3.7 \mathrm{~cm}$ long, $12-40 \mathrm{~mm}$ wide; cool season growth 0.5- $0.7 \mathrm{~cm}$ long, 4- $6 \mathrm{~mm}$ wide. Foliage color greenish-yellow in winter, light green in summer, greenish-yellow on new growth. Warm season leaves linear, straight to slightly recurved, 8 to $27 \mathrm{~mm}$ long, apex straight, acute to acuminate, attachment ascending to perpendicular, 45-90\% angle. Cool season leaves linear, straight to recurved, 1-5 mm long, apex straight, acute to acuminate, attachment ascending to perpendicular, $60-90 \%$ angle. Female strobili rare. $\mathrm{M}$ ale strobili persistent, clustered on primary shoots, proximal, 1-8 per cluster; conical, 3-7 $\mathrm{mm}$ long, 2-3 mm wide, apex acute. 
Vouchers: 166, 180, 191, 235, 238.

O bservations: Linear leaf selection distinguished by the greenish-yellow new and winter foliage.

'E legans Compacta' D allimore and Jackson (1948). D warf shrub to $0.7 \mathrm{~m}$ tall, $1.3 \mathrm{~m}$ wide, dense spreading mounded. Primary shoots 2-17 cm long; secondary shoots 9-24, $1.8-9 \mathrm{~cm}$ long, congested; tertiary shoots $0-10,0.4-5.0$ $\mathrm{cm}$ long, congested; quaternary shoots lacking; seasonal growth difference conspicuous, warm season growth 3.5$5.5 \mathrm{~cm}$ long, $12-30 \mathrm{~mm}$ wide; cool season growth $0.5-0.7 \mathrm{~cm}$ long, 4-6 mm wide. Foliage color dark green to bronze in winter, medium green in summer with terminal shoots often yellow, medium green or yellow on new growth. Warm season leaves saber, straight, (9) 11-17 $\mathrm{mm}$ long, apex straight to reflexed, acute to acuminate, attachment ascending 30$45 \%$ angle. Cool season leaves short saber, straight to recurved, apex straight, acute to acuminate, 1-5 mm long, attachment ascending to perpendicular, $60-90 \%$ angle. Female strobili rare. $M$ ale strobili persistent, proximal on primary and secondary shoots, in 1-6 globose complexes of strobili fused at the base; strobili complex 10-15 mm long, 9-13 $\mathrm{mm}$ wide, with 8-16 strobili per complex; strobilus oval, apex obtuse, 3-6 mm long, 2-3 mm wide. Vouchers: 253, 273, 282.

O bservations: 'Elegans Compacta' is confused with ' $\mathrm{N}$ ana', but distinguished by the more prostrate habit.

\section{'E legans G racilis' H ort. = 'E legans Nana'}

'E legans $\mathbf{N}$ ana' = ' $\mathrm{N}$ ana' pro parte O bservations: $\mathrm{N}$ ame applied to two distinct cultivars, misapplied here. $\mathrm{N}$ amed as variety by Knight and Perry (1850), but polynominal name was illegitimate; as cultivar by Welch (1966), but name illegitimate as published for another cultivar a year earlier.

\footnotetext{
'E legans $\mathbf{N}$ ana' [var. elegansnana Veitch; 'Elegans G racilis']. Compact shrub (juvenile $1 \mathrm{~m}$ tall, $2 \mathrm{~m}$ wide), irregular with conical upturned branches. Primary shoots perpendicular, $19-27 \mathrm{~cm}$ long; secondary shoots $10-19,1.7-12 \mathrm{~cm}$ long; tertiary shoots $0-7,1.5-6.5 \mathrm{~cm}$ long; quaternary shoots lacking; seasonal growth conspicuous; warm season growth $1.4-4.5 \mathrm{~cm}$ long, $13-49 \mathrm{~mm}$ wide; cool season growth $0.5-0.7 \mathrm{~cm}$ long, $4-6 \mathrm{~mm}$ wide. Foliage color bronze to purplish-red in winter, medium green in summer, medium green on new growth. Warm
}

season leaves linear, straight to slightly recurved, 8-25 mm long, apex inflexed, acute to acuminate, attachment perpendicular, $80-95 \%$ angle. Cool season leaves linear, straight to recurved, 1-5 mm long, apex straight, acute to acuminate, attachment ascending to perpendicular, 60-90\%angle. Female strobili rare. $M$ ale strobili persistent, oblong oval, 3-7 mm long, 2-3 mm wide, apex acute, 1-8 per cluster, proximal on primary shoots. Voucher: 277. D escribed as variety by Veitch (1881); as cultivar by D en O uden and Boom (1965).

O bservations: Confused with ' $N$ ana', but distinguished by the linear leaves. This cultivar has foliage and branching pattern similar to 'Elegans', but differs in the limbs being decumbent, growing down toward the ground and turning up distally.

'Elegans Viridis' [var. elegansviridis $\mathrm{H}$ ornibrook]. Small tree (juvenile $3 \mathrm{~m}$ tall, $1 \mathrm{~m}$ wide), columnar to narrowly conical. Primary shoots perpendicular, $19-27 \mathrm{~cm}$ long; secondary shoots $10-19,1.7-12 \mathrm{~cm}$ long; tertiary shoots 0-9, $1.5-7 \mathrm{~cm}$ long; quaternary shoots lacking; seasonal growth conspicuous; warm season growth $13-52 \mathrm{~mm}$ wide; cool season growth $0.5-$ $0.7 \mathrm{~cm}$ long, 4- $6 \mathrm{~mm}$ wide. Foliage color medium green in winter, summer, and on new growth. Warm season leaves juvenile, linear, straight to slightly recurved, 8-25 $\mathrm{mm}$ long, apex inflexed, acute to acuminate, attachment perpendicular, $80-95 \%$ angle. Cool season leaves linear, straight to recurved, 1-5 mm long, apex straight, acute to acuminate, attachment ascending to perpendicular, $60-90 \%$ angle. Female strobili rare. M ale strobili persistent, oblong oval, 3-7 mm long, 2-3 mm wide, apex acute, 1-8 per cluster, proximal on primary shoots. Voucher: 293. N amed as variety (H ornibrook, 1923); as cultivar ( $D$ en O uden and Boom, 1965).

O bservations: 'E legans V iridis' is nearly identical to 'Elegans' in morphology and growth habit, but distinguished by the persistent green winter coloration.

'Enko-sugi' Fitschen (1930) = 'Araucarioides'

'G iokomo' H ort. = 'Giokumo'

'G iokumo' H ort. ['Gyokruyu']. Compact shrub to $2 \mathrm{~m}$ tall, $1 \mathrm{~m}$ wide, conical. Primary shoot 8-31 cm long; secondary shoots 6-13, 1-14 cm long; tertiary shoots $0-6,0.8-2.5 \mathrm{~cm}$ long; quaternary shoots $0-2,0.5-1 \mathrm{~cm}$ long; seasonal growth conspicuous; warm season growth
1.5-3 cm long, $13-30 \mathrm{~mm}$ wide; cool season growth $0.5-1.1 \mathrm{~cm}$ long, $3-7 \mathrm{~mm}$ wide. Foliage color medium green in winter and summer, bluish-green on new growth. Warm season leaves saber, slightly incurved, 6-24 mm long, apex straight to inflexed, acute, attachment ascending 45$60 \%$ angle. Cool season leaves short saber, straight, 2-5 mm long, apex straight, acute, attachment ascending 45-60\% angle. Female strobili rare. M ale strobili rare. Vouchers: 177, 217.

O bservations: 'Giokomo', 'Giokuro', 'Gyo Kruya', 'Gyokruyu' and 'Gyo Kuryu' are common misspellings found in both botanic gardens and the nursery and landscape industries. Cool season growth very short. Similar to 'Black D ragon' but distinguished by broader conical form and medium green foliage.

'Giokuro' = 'Giokumo'

'Globes' H ort. ['Globus']. Compact shrub (juvenile $2 \mathrm{~m}$ tall, $1 \mathrm{~m}$ wide), pyramidal to oval. Primary shoots perpendicular, 17-34 cm long; secondary shoots $6-23,1-10 \mathrm{~cm}$ long; tertiary shoots $0-7,1-4 \mathrm{~cm}$ long; quaternary shoots lacking; seasonal growth conspicuous; warm season growth $1.4-3.7 \mathrm{~cm}$ long, $25-42 \mathrm{~mm}$ wide; cool season growth $0.4-0.8 \mathrm{~cm}$ long, 3-6 mm wide. Foliage color bronze to purplish-red in winter, medium green in summer, medium green on new growth. Warm season leaves juvenile, linear, slightly recurved to deeply recurved, $12-25 \mathrm{~mm}$ long, apex inflexed, acute to acuminate, attachment perpendicular, $80-90 \%$ angle. Cool season leaves linear, straight to recurved, 1-5 mm long, apex straight, acute to acuminate, attachment ascending to perpendicular, $60-90 \%$ angle. Female strobili rare. M ale strobili persistent, clustered on primary shoots or first secondary shoot, proximal, 2-25 per cluster, conical, 5-14 mm long, 2-5 mm wide, apex acute. Vouchers: 176, 239.

O bservations: Linear-leaved cultivar distinguished by the purplish-red summer foliage.

'G lobosa' D en O uden and Boom (1949). Compact shrub to $1.3 \mathrm{~m}$ tall, $1 \mathrm{~m}$ wide, subglobose. Primary shoot ascending, 11-19 cm long; secondary shoots $1-$ $12,0.5-14 \mathrm{~cm}$ long; tertiary shoots $0-6$, $0.5-6 \mathrm{~cm}$ long; quaternary shoots (0) 3$6,0.5-2.5 \mathrm{~cm}$ long, new growth spirally twisted; seasonal growth conspicuous; warm season growth 3-10.5 cm long, 6$11 \mathrm{~mm}$ wide; cool season growth 0.3-1.2 $\mathrm{cm}$ long, 3-6 $\mathrm{mm}$ wide. Foliage color purplish-red in winter, medium green in summer, bluish-green on new growth. 
Warm season leaves saber, straight to slightly incurved, 7-11 mm long, apex straight to inflexed, acute, attachment appressed to ascending 10-45\% angle. Cool season leaves short saber, slightly incurved, 2-4 mm long, apex straight to inflexed, acute, attachment ascending 5$45 \%$ angle. Female strobili rare. $M$ ale strobili rare. Vouchers: 223, 252, 254.

O bservations: Cool seasonal growth very short. 'Globosa' is a shorter, more compact plant than 'Globosa N ana' with which it is confused. M orphological similarity and misleading cultivar name has led to many misidentifications in both botanic gardens and the nursery and landscape industries.

'G lobosa N ana' [var. globosa nana H ornibrook; 'L obbii N ana']. Compact shrub to $2 \mathrm{~m}$ tall, $2 \mathrm{~m}$ wide, subglobose to oval. Primary shoot ascending, 3.5-12 $\mathrm{cm}$ long; secondary shoots $1-18,0.8$ to $7.2 \mathrm{~cm}$ long; tertiary shoots $0-7,0.5-3$ cm long; quaternary shoots lacking; seasonal growth conspicuous; warm season growth $0.8-9 \mathrm{~cm}$ long, 7-16 mm wide; cool season growth $0.4-1.6 \mathrm{~cm}$ long, 4-8 $\mathrm{mm}$ wide. Foliage color purplish-red in winter, medium green in summer, bluish-green on new growth. Warm season leaves saber, straight, 5-10 $\mathrm{mm}$ long, apex straight, acute to acuminate, attachment ascending 40-60\% angle. Cool season leaves short saber, straight, 2-4 mm long, apex straight to reflexed, acute to acuminate, attachment ascending $30-45 \%$ angle. Female strobili rare. $\mathrm{M}$ ale strobili rare. Vouchers: 179, 245, 259, $261,270,274$. N amed as variety (H ornibrook, 1923), as cultivar (Den O uden and Boom, 1965).

O bservations: 'G lobosa N ana' grows larger and branching is more sparse than 'Globosa' with which it is confused.

'G lobus' Tripp (1993) = 'G lobes'

'G olden E legance' = proposed name for 'E legans Aurea' as per Article 17.9 of the ICNCP.

'G raceful' = proposed name for ' $G$ racilis' as per Article 17.9 in accordance with the ICNCP.

'G racilis' Krüssmann (1972) = 'G raceful' Tree to $5 \mathrm{~m}$ tall, $2 \mathrm{~m}$ wide, broad conical to narrow pyramidal. Primary shoot $15-$ $29 \mathrm{~cm}$ long; secondary shoots 12-45, $0.5-22 \mathrm{~cm}$ long; tertiary shoots $0-31$, $0.3-8 \mathrm{~cm}$ long; quaternary shoots $0-6$, $0.3-2.5 \mathrm{~cm}$ long; seasonal growth conspicuous; warm season growth $4-8 \mathrm{~cm}$ long, 4-6 $\mathrm{mm}$ wide; cool season growth 0.8-2.8 cm long, 2-4 mm wide. Foliage color greenish-yellow in winter, summer, and on new growth. Warm season leaves short saber, straight to slightly incurved, 3-5 mm long, apex straight or inflexed, acuminate, attachment ascending 45\% angle. Cool season leaves short saber, straight to slightly incurved, $1-3 \mathrm{~mm}$ long, apex straight or inflexed, acute to acuminate, attachment appressed to ascending, 10-45\% angle. Female strobili persistent, terminal on secondary or tertiary shoots, solitary, subglobose, 8-14 $\mathrm{mm}$ long, $10-14 \mathrm{~mm}$ wide; scale-bracts 15-20, quadrangular, 5-8 mm long, 5-8 $\mathrm{mm}$ wide, apex acute, attachment cuneate to peltate; umbo straight or reflexed, acute; processes 1-3, 2-4 mm long, exserted. M ale strobili rare. Vouchers: 201, 278.

O bservations: D istinguished as tree with short saber leaves with foliage consistently greenish-yellow all year.

\section{'G ranny's R inglets' H ort. = 'Spiralis'}

'G reen Pencil' H ort. Compact shrub, adult, $2 \mathrm{~m}$ tall, $1 \mathrm{~m}$ wide, oval to ovoid. Primary shoot 4-10 cm long; secondary shoots 6-10, 0.3-5.3 cm long; tertiary shoots $0-3,0.3-3.0 \mathrm{~cm}$ long; terminal shoots clustered, often towards the apex of the crown; quaternary shoots lacking; seasonal growth conspicuous; warm season growth 1.2-1.7 cm long, 10-17 $\mathrm{mm}$ wide; cool season growth $0.3-2.6 \mathrm{~cm}$ long, 5-6 mm wide. Foliage color bronze in winter, medium green in summer, light green on new growth. Warm season leaves adult, saber, straight to slightly recurved, 6-10 mm long, apex straight, acute, attachment ascending 45-60\%angle. Cool season leaves adult, short saber, straight to slightly recurved, 1-4 mm long, apex reflexed, acute, attachment ascending $45 \%$ angle. Female strobili rare. M ale strobili rare. Vouchers: 212, 213.

O bservations: $M$ ore plants required for additional data for adequate characterization. Known from Atlanta Botanic Garden.

'G yo Kruya' H ort. = 'G iokimo'

'G yo Kuryu' H ort. = 'Giokumo'

'G yokruyu' H ort. = 'G iokumo'

'H ime-ikari-sugi' H ort. ='I kari'

'H ime-sugi' H ort. = 'Elegans'
'H ino-sugi' H ort. = 'Vilmoriniana'

'I kan-sugi' H ort. = 'I kari'

'I kar-sugi' H ort. = 'I kari'

'I kari' Kortmann (1987). [ 'H ime-ikarisugi'; 'Ikan-sugi'; 'Ikar-sugi']. Compact shrub to $1.5 \mathrm{~m}$ tall, $1.5 \mathrm{~m}$ wide, broad pyramidal to subglobose. Primary shoot 10-20 cm long; secondary shoots 9-18, pendulous, $1.2-13.8 \mathrm{~cm}$ long; tertiary shoots (0) 3-12, pendulous, $1-8.4 \mathrm{~cm}$ long; quaternary shoots $0-1,0.4-0.8 \mathrm{~cm}$ long; seasonal growth conspicuous; warm season growth $0.8-1.8 \mathrm{~cm}$ long, 7-12 $\mathrm{mm}$ wide; cool season growth (1.5) 3$11.5 \mathrm{~cm}$ long, 4-5 mm wide. Foliage color light green in winter, medium green in summer, light green on new growth. Warm season leaves short saber, straight to slightly incurved, 3-6 mm long, apex straight or reflexed, acute to acuminate, attachment ascending $45 \%$ angle. Cool seasonal leaves short saber, straight to slightly incurved, 1-3 mm long, apex straight or reflexed, acute to acuminate, attachment ascending 45\% angle. Female strobili rare. M ale strobili rare. Voucher: 199.

O bservations: Compact shrub with short saber leaves with light green new and winter foliage.

'I to-sugi' H ort. = 'Bandai-sugi’ pro parte

'I to-sugi' H ort = 'Taisho-tama' pro parte

'I to-sugi' Krüssmann (1972). N ot observed, but Welch (1979) noted similarity to 'Araucarioides'.

'J indai-sugi' [var. jindai sugi H ornibrook]. Compact shrub to small tree, to $3 \mathrm{~m}$ tall, $1.5 \mathrm{~m}$ wide, broad conical to pyramidal when mature. Primary shoot (6.5) 9-18.5 cm long; secondary shoots 6-20, $1.5-8 \mathrm{~cm}$ long, congested along axis; tertiary shoots $0-9$, $1-4.5 \mathrm{~cm}$ long, congested along axis; quaternary shoots $0-1,0.4-1 \mathrm{~cm}$ long; seasonal growth difference conspicuous; warm season growth 0.8-1.8 $(2.0) \mathrm{cm}$ long, $6-15 \mathrm{~mm}$ wide; cool season growth $0.5-1.5 \mathrm{~cm}$ long, 3-6 mm wide. Foliage color bronze in winter, dark green in summer, medium green on new growth. Warm season leaves short saber, straight to slightly incurved or slightly recurved, (4) 6-11 mm long, apex straight, acute to acuminate, attachment ascending 30-60\% angle. Cool season leaves short saber, straight, 1-3 mm long, apex straight to 
inflexed, acute, attachment appressed to ascending 5-45\% angle. Female strobili rare. $\mathrm{M}$ ale strobili persistent, terminal strobili complex on secondary and tertiary shoots, 1-12 per cluster, oblong, 2-10 $\mathrm{mm}$ long, 1.5-3 mm wide, apex obtuse; occasionally compound strobilus with 1-2 conelets. Vouchers: 205, 257, 262, 268. $\mathrm{N}$ amed as variety ( $\mathrm{H}$ ornibrook, 1923); as cultivar (D en O uden and Boom, 1965).

O bservations: This cultivar often is found mislabeled in the $U$ nited States under the name 'Bandai-sugi'. Similar to 'T aishotama', but distinguished by shorter seasonal growth and secondary shoots, and longer, oblong male strobili.

'Kilmacurragh' H ornibrook (1938). Small tree to $6 \mathrm{~m}$ tall, $2.5 \mathrm{~m}$ wide, pyramidal, trunk knotted or irregularly twisted. Primary shoots ascending, 5-22 $\mathrm{cm}$ long; secondary shoots $1-30,1-16 \mathrm{~cm}$ long, occasionally fasciated; tertiary shoots 0-20, 1-6 cm long, occasionally fasciated; quaternary shoots lacking; seasonal growth not conspicuous; nonfasciated growth 5-10 cm long, 15-28 mm wide; fasciated shoots 1-6 (8) cm long, 1-6 (10.5) $\mathrm{cm}$ wide. Foliage color bronze in winter, medium green in summer, light green on new growth. N onfasciated leaves straight saber, straight, 7-18 mm long, apex straight to reflexed, acute to acuminate, attachment ascending 45-60\% angle. Fasciated leaves straight saber, straight, 4-18 mm long, apex straight to reflexed, acute to acuminate, appressed $45 \%$ angle. Female strobili rare. $M$ ale strobili rare. Voucher: 207.

'Kilmacurragh' described by $\mathrm{H}$ ornibrook (1938) from a specimen located at Kilmacurragh, I reland, as belonging to var. cristata Bleiss

O bservations: 'Kilmacurragh' has smaller fasciated (cockscomb-like) shoots than 'Cristata'.

'Knaptonensis' [var. knaptonensisL yttel]. D warf shrub to $1 \mathrm{~m}$ tall, $0.7 \mathrm{~m}$ wide, ovoid to oval. Primary shoots ascending, $6-19.5 \mathrm{~cm}$ long; secondary shoots $9-12$, 3-13 cm long; tertiary shoots $0-7,0.5-3$ $\mathrm{cm}$ long; quaternary shoots $0-2,0.5-2$ $\mathrm{cm}$ long; pentanary shoots $0-1,0.5-0.8$ $\mathrm{cm}$ long; seasonal growth conspicuous; warm season growth $2.5-3.5 \mathrm{~cm}$ long, 9 $13 \mathrm{~mm}$ wide; cool season growth $0.9-2.0$ $\mathrm{cm}$ long, 3-5 mm wide. Foliage color bronze in winter, light green in summer, silvery-white on new growth, variegations completely engulf tertiaries and quarternary shoots or borne as irregular patches, darkening to light green. Warm season leaves short saber, slightly incurved, 2-4 mm long, apex inflexed, narrowly acuminate, attachment ascending $30-45 \%$ angle. Cool season leaves short saber, slightly incurved, 1-3 mm long, apex inflexed, narrowly acuminate, attachment ascending, 30-45\% angle. Female strobili rare. $\mathrm{M}$ ale strobili rare. Vouchers: 169, 221. D escribed as variety (L yttel, 1934); as cultivar (H ornibrook, 1938).

O bservations: D warf shrub with silverywhite new foliage. T ripp (1993) reported that hot weather causes this cultivar to revert, both in variegation color and habit, to ' $\mathrm{N}$ ana Albospica' in the southeastern U nited States. Cultivars labeled as 'Knaptonensis' in the southeastern U.S. often are misidentified as 'N ana Albospica'. ' $N$ ana Albospica' is distinguished by longer leaves and new leaves being creamy to silvery-white colored.

'Kukamiga' H ort. D warf shrub, adult, 1 $\mathrm{m}$ tall, $0.8 \mathrm{~m}$ wide, oval to ovoid. Primary shoot 4-6.5 cm long; secondary shoots $1-6,1-3.5 \mathrm{~cm}$ long; tertiary shoots $0-4$, $0.5-2.5 \mathrm{~cm}$ long; quaternary shoots lacking; seasonal growth conspicuous; warm season growth $2.5-7 \mathrm{~cm}$ long, 17$23 \mathrm{~mm}$ wide; cool season growth 0.7-1.0 $\mathrm{cm}$ long, 4-6 $\mathrm{mm}$ wide. Foliage color bronze in winter, medium green in summer, light green on new growth. Warm season leaves saber, straight to slightly incurved, 8-14 mm long, apex straight, acuminate, attachment ascending 45-60\% angle, new leaves occasionally 23 grafted. Cool season leaves short saber, straight to slightly incurved, 3-4 mm long, apex straight, acute, attachment ascending 45\% angle. Female strobili rare. M ale strobili rare. Voucher: 244.

O bservations: $M$ ore plants are required for additional data for adequate characterization. Known from Atlanta Botanical Garden.

\section{'K usari-sugi' D allimore and J ackson (1948) $=$ 'Spiraliter Falcata'}

'L ittle D iamond' L aar (1990). D warf shrub to $0.5 \mathrm{~m}$ tall, $0.5 \mathrm{~m}$ wide, globose, extremely compact. Primary shoot 5-6.5 $\mathrm{cm}$ long; secondary shoots 5-8, 0.4-25 $\mathrm{cm}$ long; tertiary shoots $0-4,0.4-1 \mathrm{~cm}$ long; quaternary shoots lacking; seasonal growth conspicuous; warm season growth 0.8-1.5 cm long, 6-7 mm wide; cool season growth $1.2-2.5 \mathrm{~cm}$ long, $3-4 \mathrm{~mm}$ wide. Foliage color medium green in winter, dark green in summer, bluishgreen on new growth. Warm season leaves saber, slightly incurved, (4) 6-10 $\mathrm{mm}$ long, apex inflexed, acute to acuminate, attachment ascending 45\% angle. Cool season leaves short saber, slightly incurved to deeply incurved, 2-3 $\mathrm{mm}$ long, apex inflexed, acute, attach- ment appressed 5-20\% angle. Female strobili rare. M ale strobili rare. V oucher: 219.

O bservations: A diminutive form of 'Bloomers Witches Broom', differing with narrower shoots, smaller habit, and slower growth.

'Littleworth D warf' Welch (1991) ['Littleworth Gnome'; 'L ittleworth Gnom']. D warf shrub, adult, $0.7 \mathrm{~m}$ tall, $0.7 \mathrm{~m}$ wide, subglobose. Primary shoot 7$10 \mathrm{~cm}$ long; secondary shoots 3-6, 2.3$4.8 \mathrm{~cm}$ long; tertiary shoots $0-1,0.8-1$ $\mathrm{cm}$ long; quaternary shoots lacking, new shoots spirally twisted; seasonal growth conspicuous; warm season growth 0.8-2 cm long, 8-12 mm wide; cool season growth $0.4-0.8 \mathrm{~cm}$ long, $3-7 \mathrm{~mm}$ wide. Foliage color medium green in summer, bronze in winter, light green on new growth. Warm seasonal leaves saber, straight to slightly incurved, 6-9 mm long, apex straight to inflexed, acuminate, attachment ascending $45 \%$ angle. Cool season leaves adult, short saber, slightly to deeply incurved, 2-5 mm long, apex inflexed, acuminate, attachment ascending $45 \%$ angle. Female strobili rare. $\mathrm{M}$ ale strobili rare. Voucher: 215.

O bservations: $M$ ore plants required for additional data for adequate characterization.

'Littleworth G nom' H ort. = 'L ittleworth Dwarf'

\section{'Littleworth G nome' H ort. =} 'Littleworth D warf'

'Lobbii' [C ryptomeria lobbiana Billain]. Tree to $30 \mathrm{~m}$ tall, $5 \mathrm{~m}$ wide, conical, congested proliferation of secondary, tertiary, and quaternary shoots at terminal end of major limbs. Primary shoot ascending, 20-55 cm long; secondary shoots 8-25, pendulous, $0.4-23 \mathrm{~cm}$ long, inner dead shoots persistent; tertiary shoots 0-24, pendulous, $0.4-6 \mathrm{~cm}$ long, inner dead shoots persistent; quarternary shoots $0-5$, pendulous, $0.5-0.7 \mathrm{~cm}$ long, inner dead shoots persistent; seasonal growth conspicuous; warm season growth 2-17.5 cm long, 8-18 mm wide; cool season growth $0.5-5 \mathrm{~cm}$ long, 4-6 mm wide. Foliage color brown in winter, medium green in summer, light green on new growth. Warm season leaves saber, slightly incurved, 8-20 mm long, apex straight to inflexed, acute to acuminate, attachment ascending 20-45\% angle. Cool season leaves short saber, straight to slightly incurved, 2-3 mm long, apex straight, acute, attachment ascending 45\% angle. Female strobili persistent 2 years, terminal on secondary or tertiary shoots, solitary, subglobose, 14-16 mm long, 11- 
$13 \mathrm{~mm}$ wide; scale-bracts $15-30,3-5 \mathrm{~mm}$ long, 3-5 mm wide, quadrangular, apex acute, attachment cuneate to peltate; umbo acute, reflexed; processes 3-6, 2-3 $\mathrm{mm}$ long, exserted. M ale strobili persistent, terminal spikelike clusters on secondary and tertiary shoots, 3-33 per cluster, ovoid, 3-5 mm long, 2-3 mm wide, apex obtuse. Vouchers: 192, 249, 250. D escribed as species (Billain, 1853); as cultivar (D allimore and Jackson, 1948).

O bservations: This selection is virtually identical to members of the typical species in most characters when not subjected to consistently cold winters. Then, 'tufting' of secondary shoots is more prevalent.

'L obbii N ana' H illier (1964) = 'G lobosa N ana'

'Lobbii N ana' D allimore and J ackson (1948) $=$ 'N ana'

'Lobbii N ana Aurea' Tripp (1993) = 'Lobbs Golden D warf'. Compact shrub to $1.5 \mathrm{~m}$ tall, $1-1.5 \mathrm{~m}$ wide, ovoid to pyramidal. Primary shoot ascending, $16-28 \mathrm{~cm}$ long; secondary shoots $11-21,0.5-12.1 \mathrm{~cm}$ long; tertiary shoots $0-13,0.5-4.9 \mathrm{~cm}$ long; quaternary shoots $0-3,0.5-0.7$; seasonal growth conspicuous; warm season growth 1-2.5 cm long, 11-20 mm wide; cool season growth (3) 4-12 cm long, 3-7 mm wide. Foliage color greenish-yellow in winter, summer, and on new growth. Warm season leaves saber, straight to slightly recurved, 5-9 mm long, apex straight, acute, attachment ascending $45-60 \%$ angle. Cool season leaves short saber, straight, 2-4 mm long, apex straight or reflexed, acute to acuminate, attachment ascending $45 \%$ angle. Female strobili rare. M ale strobili persistent, terminal spikelike clusters on secondary and tertiary shoots attached to trunk, 2-18 per cluster, ovoid, 4-5 mm long, 2-3 mm wide, apex acute. Vouchers: 197, 204, 218.

O bservations: Shrub with greenish-yellow foliage all year.

'L obbs G olden D warf' = proposed name for 'Lobbii N ana Aurea' in accordance with Article 17.9 of the ICN CP.

'M ajiro-sugi' $\mathrm{H}$ ort. = 'N ana Albospicata'

'M anhismi-sugi' $\mathrm{H}$ ort. = 'M onstrosa

$N$ ana'

'M ankichi' $\mathrm{H}$ ort. = 'M onstrosa $\mathrm{N}$ ana'

‘M ejero-sugi' H ort. ='N ana Albospicata;
'M ejiro-sugi' H ort. ='N ana Albospicata'

'Monstrosa' Beissner and Fitschen (1930). Small tree 4-5 m tall, 1-3 m wide, irregular. Primary shoot ascending, 8-42 cm long; secondary shoots $0.3-18 \mathrm{~cm}$ long, warm season only $17-$ 20 , cool season only $20-70$; tertiary shoots $0-20,0.3-18 \mathrm{~cm}$ long; quaternary shoots $0-5,0.3-1 \mathrm{~cm}$ long; seasonal growth conspicuous, primarily cool season; warm season growth (1) 4$36 \mathrm{~cm}$ long, 9-17 mm wide; cool season growth $0.5-2.5 \mathrm{~cm}$ long, 3-7 $\mathrm{mm}$ wide. Foliage color bronze in winter, medium green in summer, medium green on new growth. Warm seasonal leaves saber, straight, 7-20 mm long, apex straight or reflexed, acute to acuminate, attachment ascending 20$45 \%$ angle. Cool season leaves short saber, straight to slightly incurved, 2-4 $\mathrm{mm}$ long, apex straight, acute, attachment ascending $45-75 \%$ angle. Female strobili rare. Male strobili persistent, terminal spikelike clusters on secondary and tertiary shoots, 1-13 per cluster, ovoid, 3-8 mm long, 2-3 mm wide, apex obtuse. Vouchers: 184, 190, 294.

Observations: Tree with irregular crown, distinguished from the dwarf selection by longer primary shoots and warm season growth, and bronze winter foliage color.

'M onstrosa $\mathbf{N}$ ana' [var. monstrosa nana H ornibrook; 'M anhismi-ugi'; 'M ankichi']. Compact shrub to $3 \mathrm{~m}$ tall, 0.6-1 m wide, crown irregular. Primary shoot 8.5-11 cm long; secondary shoots 1-4 cm long, warm seasonal only 8-20, cool season only 18-24; tertiary shoots $0-8,0.4-1.3 \mathrm{~cm}$ long; quaternary shoots $0-3,0.2-0.8 \mathrm{~cm}$ long; seasonal growth conspicuous; warm season growth (1) 2-3.5 (5) cm long, 9-14 $\mathrm{mm}$ wide; cool season growth $0.5-1.7$ $\mathrm{cm}$ long, 3-6 mm wide. Foliage color medium green in winter, summer, and on new growth. Warm season leaves saber, straight to slightly incurved, 8-15 $\mathrm{mm}$ long, apex straight, acute to acuminate, attachment ascending 45\% angle. Cool season leaves short saber, straight to slightly incurved, 1-3 mm long, apex straight, acute. Female strobili rare. M ale strobili rare. Vouchers: $231,248,269$. D escribed as variety (H ornibrook, 1938); as cultivar (D allimore and J ackson, 1948).

O bservations: ' $\mathrm{M}$ onstrosa $\mathrm{N}$ ana' has similar foliage but a smaller habit and less vigorous growth than 'M onstrosa'.

'N ana' = 'K nights D warf' [var. elegans nana Knight and Perry; 'Elegans N ana' Welch (1966); 'Lobbii N ana']. Compact shrub to $2.5 \mathrm{~m}$ tall, $2 \mathrm{~m}$ wide, broad pyramidal to subglobose. Primary shoots 2-11 cm long; secondary shoots 15-24, $1.8-6.5 \mathrm{~cm}$ long, congested, pendulous; tertiary shoots $0-17,0.4-5.0 \mathrm{~cm}$ long, congested, pendulous; quaternary shoots lacking; seasonal growth difference conspicuous; warm season growth (2.5) $3.5-7.5 \mathrm{~cm}$ long, $12-30 \mathrm{~mm}$ wide; cool season growth $0.5-0.7 \mathrm{~cm}$ long, 4-6 mm wide. Foliage color bronze to purplish-red in winter, medium green in summer, medium green on new growth. Warm season leaves straight saber, straight, (9) 11-17 mm long, apex straight, acute to acuminate, attachment ascending, 30$45 \%$ angle. Cool season leaves short saber straight to recurved, 1-5 mm long, apex straight, acute to acuminate, attachment ascending to perpendicular, $60-90 \%$ angle. Female strobili rare. M ale strobili persistent, terminal on secondary and tertiary shoots located on the upper portion of older plants, in 1-3 globose complexes of strobili fused at the base; strobili complex, 10-15 mm long, 9-13 $\mathrm{mm}$ wide, with 8-16 strobili per complex; strobilus oval, apex obtuse, 3-6 mm long, 2-3 mm wide. Vouchers: 200, 286, 290. $D$ escribed as variety (Knight and Perry, 1850); as cultivar (Welch, 1991).

O bservations: ' $\mathrm{N}$ ana' is confused often with 'Elegans $\mathrm{N}$ ana', which is distinguished as a compact shrub with linear leaves and conical, solitary, male strobili. ' $\mathrm{N}$ ana' has straight saberlike leaves with ovoid stobili arranged in a strobili complex. Welch (1991) noted that immature ' $N$ ana' cultivars are misidentified often as 'L obbii $\mathrm{N}$ ana'.

' $\mathrm{N}$ ana Albospica' D en Ouden and Boom (1965) $=$ 'D warf Whitetip' ['M ajiro-sugi' $\mathrm{H}$ ort.; 'M ejero-sugi' H ort.; 'M ejiro-sugi' $\mathrm{H}$ ort.; 'O kina-sugi' H ort.]. Compact shrub to $2 \mathrm{~m}$ tall, $1 \mathrm{~m}$ wide, ovoid to oval. Primary shoots ascending, (6.5) 9 to 26 $\mathrm{cm}$ long, secondary shoots 6 to 21,3 to $11 \mathrm{~cm}$ long, tertiary shoots 0 to $14,1.5$ to $9.5 \mathrm{~cm}$ long, quarternary shoots 0 to 8 , 0.8 to $4.5 \mathrm{~cm}$ long; seasonal growth conspicuous; warm seasonal growth 2.5 to $4.2 \mathrm{~cm}$ long, 9 to $13 \mathrm{~mm}$ wide; cool seasonal growth 0.8 to $2.7 \mathrm{~cm}$ long, 3 to $5 \mathrm{~mm}$ wide. Foliage color bronze in winter, light green in summer, creamy to silvery-white on new growth, variegations completely engulf tertiaries and quarternary shoots or as irregular patches darkening to pale green shoot. Warm seasonal leaves short saberlike, straight to slightly incurved, many twisted halfway around stem, counterclockwise and clockwise, (5) 7 to $11 \mathrm{~mm}$ long, apex straight, acute, attachment ascending 30 to $45 \%$ angle. Cool seasonal leaves short 
saberlike, slightly incurved, many twisted halfway around stem, counterclockwise and clockwise, 1 to $3 \mathrm{~mm}$ long, apex inflexed, acute, attachment ascending 30 to $45 \%$ angle. Female strobili rare. $\mathrm{M}$ ale strobili rare. Vouchers: \#165,185, 222, 256.

O bservations: This cultivar is the creamy to silvery-white variegated clone found commonly in the U nited States. 'Knaptonensis' differs with exclusively silvery-white variegations, smaller habit, and shorter leaves.

'N ational Arboretum Witches Broom' Tripp (1993) ='Bloomers Witches Bloom'

'O gon-sugi' H ort. = 'Aurea'

'O kina-sugi' $\mathrm{H}$ ort. = 'N ana Albospicata'

'O sakatama' H ort. = 'Vilmoriniana'

'O saka-tama-sugi' H ort. =

'Vilmoriniana'

'Pomona' H ort. D warf shrub (juvenile 3 $\mathrm{m}$ tall, $1.5 \mathrm{~m}$ wide), ovoid. Primary shoots green, $6.5-7.5 \mathrm{~cm}$ long; secondary shoots 6-9, 0.4-5.5 cm long; tertiary shoots $1-5$, $0.4-2.8 \mathrm{~cm}$ long; quaternary shoots $0-3$, $0.4-1.5 \mathrm{~cm}$; seasonal growth not conspicuous, 3-8 mm wide. Foliage color bluish-green in winter, summer, and on new growth. Leaves linear, straight, 3-7 $\mathrm{mm}$ long, apex straight, acute, attachment ascending $45^{\circ}$ angle. Female strobili rare. M ale strobili rare. Voucher: 225.

O bservations: D warf shrub with linear leaves and bluish-green foliage all year.

'Pygmaea' H ort. = 'Vilmoriniana'

'R asen-sugi' H ort. = 'Spiralis'

'Rasen-sugi' Grootendorst (1977) = 'Spiraliter Falcata'

'Rein's D ense J ade' H ort. = 'Bloomers Witches Bloom'

'Sekka-sugi' H ort. = 'C ristata'

'Sekkan' H ort. ['Sekkan-sugi']. T ree to $30 \mathrm{~m}$ tall, $5 \mathrm{~m}$ wide, broad conical to narrow pyramidal, central leader strong. Primary shoot 10-39.5 cm long; secondary shoots 7-22, 0.6-25 cm long; tertiary shoots $0-16,0.6-15 \mathrm{~cm}$ long; quaternary shoots $0-1,0.6-1 \mathrm{~cm}$ long; seasonal growth conspicuous; warm season growth 2.5-10 cm long, 13-35 mm wide; cool season growth $0.5-3.5 \mathrm{~cm}$ long, 4-10 $\mathrm{mm}$ wide. Foliage color light green in winter, light green to greenish-yellow in summer, yellow on new growth becoming darker with first warm night temperatures. Warm season leaves saber, straight to slightly incurved, 8-20 mm long, apex straight, acute to acuminate, attachment ascending, $30-60^{\circ}$ angle. Cool season leaves short saber, straight, 2-6 mm long, apex straight, acute to acuminate, attachment ascending $45^{\circ}$ angle. Female strobili persistent, terminal on secondary or tertiary shoots, solitary, subglobose, 11-20 mm long, 12-22 mm wide; scalebracts 15-20, attachment cuneate to peltate, quadrangular, 5-8 mm long, 5-8 $\mathrm{mm}$ wide, apex acute; umbo straight or reflexed, acute; processes 3-6, 4-6 mm long, exserted. M ale strobili persistent, terminal spikelike clusters on secondary and tertiary shoots, 1-27 per cluster, oval, 3-8 mm long, 2-4 mm wide, apex obtuse. Vouchers: 164, 175, 187, 206, 233, 276.

O bservations: tree with yellow new seasonal growth. 'Sekkan' differs from 'Aurea' by a longer duration of the yellow pigmentation on new growth and a more persistent greenish-yellow coloration throughout the year.

'Sekkwia-sugi' H ort. = 'C ristata'

'Spiralis' [ C ryptomeria japonica spiralis Siebold and Zuccarini; 'G ranny's Ringlets'; 'Rasen-sugi']. T ree to $9 \mathrm{~m}$ tall, $3 \mathrm{~m}$ wide, narrow pyramidal to broad conical. Primary shoot $12-35 \mathrm{~cm}$ long; secondary shoots $6-10,0.5-10 \mathrm{~cm}$ long; tertiary shoots $0-2,0.5-4.5 \mathrm{~cm}$ long; quaternary shoots lacking; seasonal growth difference conspicuous; warm season growth 2-4 cm long, 9-14 mm wide; cool season growth $1.1-4.6 \mathrm{~cm}$ long, 3-5 mm wide. Foliage color medium green in winter, summer, and on new growth. Warm season leaves saber, deeply incurved to spirally twisted, many twisted more than halfway around stem, 10-20 mm long, apex straight, acuminate, attachment ascending 45\% angle. Cool season leaves saber, deeply incurved to spirally twisted, many twisted more than halfway around stem, 2-4 mm long, apex straight or reflexed, acuminate, attachment ascending 30-45\% angle. Female strobili rare. M ale strobili rare. Vouchers: 194, 246, 258. N amed as variety (Siebold and Zuccarini, 1844); as cultivar (Siebold, 1861).

O bservations: Cultivar with leaves spirally arranged around the twig like stands of a rope. 'Spiralis' is confused often with 'Spiraliter Falcata', a compact shrub with much thinner and longer shoots that often are more congested and twisted to form incomplete circles.

'Spiralis E longata' H ort. = 'Spiraliter Falcata'

'Spiraliter Falcata' Carrière (1876) [f. torta H ort.; 'Kusari-sugi'; 'Rasen-sugi'; 'Spiralis Elongata'; 'Yore-sugi']. Compact shrub to $1.5 \mathrm{~m}$ tall, $1.7 \mathrm{~m}$ wide, irregular. Primary shoot $11-32 \mathrm{~cm}$ long; secondary shoots 4-16, 0.7-27 cm long; tertiary shoots $0-16,0.7-16 \mathrm{~cm}$ long; quaternary shoots 0-2, 0.5-2.5 cm; seasonal growth difference conspicuous; warm season growth 4-7 cm long, 4-9 mm wide; cool season growth $1.1-7.5 \mathrm{~cm}$ long, 3-6 mm wide. Foliage color light green in winter, summer, and on new growth. Warm season leaves saber, straight, deeply incurved, or spirally twisted, many twisted more than halfway around stem, 9-15 $\mathrm{mm}$ long, apex straight or reflexed, acuminate, attachment ascending 30-45\% angle. Cool season leaves short saber, deeply incurved or spirally twisted, many twisted more than halfway around stem, 2-5 mm long, apex straight or reflexed, acuminate, attachment ascending 30-45\% angle. Female strobili rare. $\mathrm{M}$ ale strobili rare. Vouchers: 168, 183, 226, 265, 279.

O bservations: Shrub with light green foliage all year, the leaves spirally twisted, often halfway around twig.

'T aisho-sugi' H ort. = 'T aisho-tama'

'Taisho-tama' H ort. ['I to-sugi'; 'T aishosugi'; 'Taishotama-sugi'; 'T aisho

Tamasugi']. Compact shrub to small tree, to $4 \mathrm{~m}$ tall, $2 \mathrm{~m}$ wide in 20 years, crown irregular. Primary shoots upturned, (8) 10.5-29.5 cm long; secondary shoots 9-25, $0.6-10 \mathrm{~cm}$ long; tertiary shoots $0-14$, nodding, $0.5-6 \mathrm{~cm}$ long; quaternary shoots 0-6, nodding, $0.4-2 \mathrm{~cm}$ long; seasonal growth difference conspicuous; warm season growth (1.5) 1.9-6 cm long, 5-15 mm wide; cool season growth 1-2.5 (3) cm long, 3-4 $\mathrm{mm}$ wide. Foliage color brown in winter, dark green in summer, medium green on new growth. Warm season leaves short saber, straight to slightly incurved, (4) 6-10 mm long, apex straight or inflexed, acute to acuminate, attachment ascending $45 \%$ angle. Cool season leaves short saber, straight to slightly incurved, 1-3 mm long, apex inflexed, acute to acuminate, attachment ascending $45 \%$ angle. Female strobili rare. M ale strobili persistent, terminal spikelike clusters on tertiary shoots, 1-13 per cluster, ovoid, 3-6 mm long, 2-3 mm wide, apex obtuse. Vouchers: 174, 216, 255, 281. 
O bservations: Similar to 'J indai-sugi', distinguished by longer seasonal shoots and secondary shoots, and shorter ovoid male strobili.

'T aishotama-sugi' H ort. = 'T aisho-tama'

'T aisho Tamasugi' H ort. = 'T aishotama'

'T ansu' (H ort.) Welch (1991)

['Yatsabusa'; 'Yatsubusa']. D warf shrub to $0.5 \mathrm{~m}$ tall, $0.5 \mathrm{~m}$ wide, irregular, extremely compact. Primary shoot $5-7.5 \mathrm{~cm}$ long; secondary shoots 12-16, 1.5-4.5 $\mathrm{cm}$ long; tertiary shoots $0-7,0.4-2.5 \mathrm{~cm}$ long; quaternary shoots lacking; seasonal growth conspicuous; warm season growth 0.9-2 cm long, 9-14 mm wide; cool season growth $0.4-1 \mathrm{~cm}$ long, $4-5 \mathrm{~mm}$ wide. Foliage color dark green in winter, dark green in summer, medium green on new growth. Warm season leaves straight saber, straight, 5-10 mm long, apex straight or reflexed, acuminate, attachment ascending 45-60\% angle. Cool season leaves short saber, straight to slightly recurved, 1-3 mm long, apex reflexed, attachment ascending $45 \%$ angle acute. Female strobili rare. M ale strobili rare. Voucher: 224.

O bservations: D warf shrub with straight saber leaves similar to 'Elegans Compacta', but with more green in foliage seasonally.

'Tenzan' (H ort.) Welch (1991) ['Tenzan Yatsabusa'; 'Yatsabusa'; 'Yatsubusa']. D warf shrub to $20 \mathrm{~cm}$ tall, $30 \mathrm{~cm}$ wide, mounded, extremely compact. Primary shoot $2.5-5 \mathrm{~cm}$ long; secondary shoots $5-13,0.4-3.5 \mathrm{~cm}$ long; tertiary shoots $0-7,0.4-1 \mathrm{~cm}$ long; quaternary shoots $0-1$, to $0.4 \mathrm{~cm}$ long; seasonal growth conspicuous; warm season growth $0.5-1.5 \mathrm{~cm}$ long, 7-25 mm wide; cool season growth $0.5-1.4 \mathrm{~cm}$ long, 3-6 $\mathrm{mm}$ wide. Foliage color dark green in winter, medium green in summer, bluishgreen on new growth. Warm season leaves saber, straight to slightly incurved, 6-12 $\mathrm{mm}$ long, apex straight or inflexed, acuminate, attachment ascending 45-90\%angle. Cool season leaves short saber, straight to slightly incurved, 2-4 mm long, apex straight, acute to acuminate, attachment subperpendicular, 75-85\%angle. Female strobili rare. M ale strobili rare. Vouchers: 209, 242.

O bservations: Smallest of the shrubs with bluish-green new foliage and diminitive cool season growth.

'Tenzan Yatsabusa' (H ort.) Tripp (1993) = 'T enzan'
'Vilmoriniana' H ornibrook (1924) ['H ino-sugi'; ' 'O saka-tama'; ' $O$ saka-tamasugi'; 'Pygmaea']. D warf shrub to $1 \mathrm{~m}$ tall, $0.8 \mathrm{~m}$ wide, subglobose. Primary shoot 3.5-8 (12) cm long; secondary shoots 1-18, 0.8-7.2 cm long, terminal ones nodding and exserted $2-3 \mathrm{~cm}$ beyond shrub margin; tertiary shoots $0-7$, $0.5-3 \mathrm{~cm}$ long, terminal ones nodding and exserted $2-3 \mathrm{~cm}$ beyond shrub margin; quaternary shoots lacking; seasonal growth conspicuous; warm seasonal growth $0.8-9 \mathrm{~cm}$ long, 7-16 mm wide; cool season growth $0.4-2.3 \mathrm{~cm}$ long, 4-8 $\mathrm{mm}$ wide. Foliage color bronze in winter, dark green in summer, medium green on new growth. Warm season leaves short saber, straight, (5) 6-10 mm long, apex straight, acute to acuminate, attachment ascending 40-60 angle. Cool season leaves short saber, straight, 2-4 $\mathrm{mm}$ long, apex straight or reflexed, acute to acuminate, attachment ascending 30$45 \%$ angle. Female strobili rare. $M$ ale strobili rare. Vouchers: 173, 196, 241, 251, 260, 264, 271275.

O bservations: 'Vilmoriniana' is confused often with 'Compressa' which is narrower and has terminal shoots that are not exserted or nodding, and linear warm season leaves.

'Yataduta' H ort. Compact shrub to $3 \mathrm{~m}$ tall, $1 \mathrm{~m}$ wide, globose. Primary shoot 9 $14.5 \mathrm{~cm}$ long; secondary shoots 8-11, 1-6 $\mathrm{cm}$ long; tertiary shoots $0-2,1-2.7 \mathrm{~cm}$ long; quaternary shoots lacking; seasonal growth conspicuous; warm season growth $1.5-2.0 \mathrm{~cm}$ long, 6-9 mm wide; cool season growth 1.4-2.5 cm long, 3-4 mm wide. Foliage color bronze in winter, dark green in summer, medium green on new growth. Warm season leaves short saber, straight to slightly incurved, 4-6 mm long, apex straight, acuminate, attachment ascending $45 \%$ angle. Cool season leaves short saber, straight to slightly incurved, 2-3 mm long, apex straight or rarely inflexed, acute, attachment ascending 45\% angle. Female strobili rare. $\mathrm{M}$ ale strobili rare. Voucher: 220.

Observations: Compact shrub with short saber leaves similar to 'I kari', distinguished by more green in foliage color all year and shorter cool season growth.

'Yatsabusa' H ort. = 'Tansu' pro parte, 'Tenzan' pro parte

'Yatsubusa' H ort. = 'T ansu' pro parte, 'Tenzan' pro parte, 'Yokohama' pro parte

'Yenko-sugi' D allimore and Jacksson (1948) $=$ 'Araucarioides'
'Yokohama' (H ort.) L aar (1983). ['Yatsubusa']. D warf shrub to $0.5 \mathrm{~m}$ tall, $0.3 \mathrm{~m}$ wide, extremely compact, crown irregular. Primary shoot 3-5 cm long; secondary shoots $0.3-3 \mathrm{~cm}$ long, warm season only $4-8$, cool season only $9-20$; tertiary shoots $0-4,0.3-1 \mathrm{~cm}$ long; quaternary shoots lacking; seasonal growth conspicuous; warm season growth 0.9-1.4 cm long, 9-18 $\mathrm{mm}$ wide; cool season growth $0.3-1 \mathrm{~cm}$ long, 3-4 mm wide. Foliage color medium green in summer, light green on new growth. Warm season leaves saber, straight to slightly incurved, 6-9 mm long, apex inflexed to reflexed, acuminate, attachment ascending 45-90\% angle. Cool season leaves short saber, straight, occasionally twisted around shoot, 2-3 $\mathrm{mm}$ long, apex straight, acute, attachment ascending $45 \%$ angle. Female strobili rare. M ale strobili rare. Voucher: 228.

O bservations: D warf shrub with irregular crown bearing shorter primary shoots and warm season leaves, and fewer secondary shoots of those cultivars with irregular crowns.

'Yore-sugi' H ort. = 'Spiraliter Falcata'

'Yoshino' Yokohama N ursery (1923). Tree to $30 \mathrm{~m}$ tall, $5 \mathrm{~m}$ wide, conical. Primary shoot 16-46 cm long; secondary shoots 5$16,0.4-31 \mathrm{~cm}$ long; tertiary shoots $0-25$, $0.4-18 \mathrm{~cm}$ long; quaternary shoots $0-7$, $0.4-5 \mathrm{~cm}$ long; dead shoots persistent; seasonal growth conspicuous; warm season growth 3-11 cm long, 7-15 mm wide; cool season growth 1-4 cm long, 3-5 mm wide. Foliage color bronze in winter, medium green in summer, light to bluish-green on new growth. Warm season leaves saber, slightly incurved, 6-14 mm long, apex inflexed, acute to acuminate, attachment ascending 30-45\%angle. Cool season leaves short saber, straight to slightly incurved, 2-3 $\mathrm{mm}$ long, apex straight or inflexed, acute to acuminate, attachment ascending $45 \%$ angle. Female strobili persistent, terminal on secondary or tertiary shoots, solitary, subglobose, 9-22 mm long, 14-20 mm wide; scale-bracts 15-20, quadrangular, 4-7 $\mathrm{mm}$ long and 4-8 $\mathrm{mm}$ wide, apex acute, attachment cuneate to peltate; umbo reflexed; processes 3-5, 2-3 mm long, exserted. M ale strobili persistent, terminal spikelike clusters on secondary, tertiary, and quarternary shoots, occasionally as pairs fused at the base, 4-43 per cluster, ovoid, 5$8 \mathrm{~mm}$ long, 2-3 mm wide, apex obtuse. Vouchers: 167, 211, 266, 283, 295.

O bservations. The growth form typically is six times taller than wide. The dead shoots persist, but the duration period appears shorter than in other selections of the species. 
Fig. 2. A key to selected cultivars of Cryptomeria japonica. M easurements cited are in metric units $(25.4 \mathrm{~mm}=2.54 \mathrm{~cm}=1$. 0 inch; $0.3 \mathrm{~m}=\mathbf{1 . 0} \mathrm{ft}$ ) and standard taxonomic format (number in parenthesis is measurement seldom encountered; $x-y$ is common measurement range).

1a. Linear leaves present; saber leaves lacking, or rarely found on cool season growth ....................................................... 2

1b. Linear leaves absent; saber leaves present on warm and cool season growth .............................................................8

2a. Warm season shoot 3-8 mm wide; warm season leaves 3-7 mm long, straight, ascending to $45^{\circ}$ angle, apex straight; quaternary shoots present to lacking; foliage bluish-green; dwarf shrub ........................................................................ 'Pomona'

2b. Warm season shoot 12-52 mm wide; warm season leaves 8-27 mm long, recurved to straight, ascending to perpendicular at 60$90^{\circ}$, apex inflexed or reflexed; quaternary shoots lacking; foliage green to bronze/ purplish-red (rarely bluish-green when new); compact shrub to narrowly columnar, small tree

3a. Cool season leaf short saber, apex reflexed; cool season growth 7-18 mm long; new foliage bluish-green becoming dark green in summer; ovoid dwarf shrub $1 \mathrm{~m}$ tall $\times 0.5 \mathrm{~m}$ wide

'Compressa'

3b. Cool season leaf linear, apex inflexed to straight; cool season growth 4-8 $\mathrm{mm}$ long; new and summer foliage light to medium green; compact shrub (1-2 m tall $\times 1-2 \mathrm{~m}$ wide) or small tree

4a. Plant twice broader than tall; crown irregular with limbs decumbent and conical upturned branches; compact shrub to $1 \mathrm{~m}$ tall

'E legans N ana'

4b. Plant 2-3 times taller than wide; crown regular, ovoid or columnar, with branches spreading; compact shrub to small tree ...... 5

5a. Warm season leaves slightly to deeply recurved, shorter ones 12-15 mm long; warm season shoots broader, 25-42 mm wide; crown pyramidal-oval; compact shrub

'G lobes'

5b. Warm season leaves straight to slightly recurved, shorter ones 8-11 mm long; warm season shoots narrower, 12-52 mm wide; crown narrowly columnar; small tree

6

6a. Cool season leaf apex straight; warm season leaf attachment ascending to perpendicular at 45-90'; twigs and leaves greenishyellow in summer and winter

'E legans Aurea'

6b. Cool season leaf apex inflexed; warm season leaf attachment perpendicular at 80-90'; twigs and leaves light to medium green in summer to bronze/ purplish-red in winter

7a. T wigs and leaves light to medium green in winter

'E legans Viridis'

7b. T wigs bronze with leaves bronze to purplish-red in winter

'E legans'

8a. Secondary and tertiary shoots with fasciations (cockcomb-like), shootsexpanded laterally and appearing fanlike; cool season leaves 4-18 mm long; trunks irregularly twisted

8b. Secondary and tertiary shoots lacking fasciations; cool season leaves 1-6 mm long; trunks straight

9a. Fasciations large, 7-15.5 cm long, 2.5-10.5 cm wide; fasciated shoots $0-1$ per primary shoot; leaf shaft straight to incurved, apex straight to inflexed

'C ristata'

9b. Fasciations small, 1-8 cm long, 1-6 (10) cm wide; fasciated shoots 1-2 per primary shoot; leaf shaft straight, apex straight to reflexed

'Kilmacurragh'

10a. Primary shoots with secondary shoots lacking or clustered near the apex and appearing tuft-liked; quaternary shoots lacking; tree growth form with female strobili typically lacking

10b. Primary shoot with secondary shoots distributed along axis; quaternary shoots present, 1-8, occasionally lacking; tree growth form with female strobili present, or dwarf to large shrub growth form

11a. Secondary shoots few $(0-10)$ and elongate, $7-35 \mathrm{~cm}$ long, spaced more proportionally along primary shoot; warm season leaves 7-14 mm long; winter color brown

'A raucarioides'

11b. Secondary shoots many (15-27) and short, 1.5-6 cm long, clustered primarily at the primary shoot apex; warm season leaves 5$7 \mathrm{~mm}$ long; winter color purplish-red

'D acrydioides'

12a. Warm season leaves short saber-like, 2-6 mm long; cool season leaves 1-3 mm long; majority of growth cool season ......... 13

12b. Warm season leaves saber-like, (5) 6-24 mm long; cool season leaves (1) 2-6 mm long; majority of growth warm season ... 16

13a. Warm season growth 4-8 cm long, 4-6 mm wide; foliage greenish-yellow all year; secondary shoots (12) 18-45; tree'G racilis'

13b. Warm season growth 1-3.5 cm long, 6-13 mm wide; foliage green to bronze (occasionally silvery-white on new growth); secondary shoots 8-18; shrub

14a. N ew foliage silvery-white; warm season growth $2.5-3.5 \mathrm{~cm}$ long; cool season growth $0.9-2 \mathrm{~cm}$ long; leaf apex inflexed; dwarf shrub, to $1 \mathrm{~m}$ tall

'Knaptonensis'

14b. $\mathrm{N}$ ew foliage green; warm season growth 1-2 cm long; cool season growth 1.5-11.5 cm long; leaf apex straight to slightly reflexed; compact shrub, 1-3 m tall

15a. Tertiary shoots 3-12; cool season growth 3-11.5 cm long, 4-5 mm wide; new growth and winter color light green, medium green in summer 
Fig. 2. Continued.

15b. Tertiary shoots 0-2; cool season growth $1.4-2.5 \mathrm{~cm}$ long, 3-4 mm wide; new growth medium green becoming dark green in summer and bronze in the winter

16a. Warm season leaves straight saber-like with a straight shaft and apex; male strobili oval, clustered in a globular strobili complex or arranged in a spikelike cluster when present .....

16b. Warm season leaves saber-like with the shaft, apex or both exhibiting some curvature; male strobili conical, oblong or ovoid, simple or with a primary strobilus and two conelets fused basally, when present

17a. Tree; cool season leaves straight; new growth yellow; quaternary shoots occasionally present, to $6 \mathrm{~mm}$ long ..................... 18

17b. Shrub; cool season leaves straight to slightly recurved; new growth medium green or greenish-yellow; quaternary shoots lacking

18a. Foliage slowly greening, becoming greenish-yellow to light green in summer, light green in winter; immature female cones bluish-green; seasonal growth more robust, warm season growth to $10 \mathrm{~cm}$ long and $35 \mathrm{~mm}$ wide, cool season growth to $3.5 \mathrm{~cm}$ long

'Sekkan'

18b. Foliage quickly greening, becoming light to medium green in summer and bronze in winter; immature female cones greenishyellow; seasonal growth less, warm season growth to $4.5 \mathrm{~cm}$ long and $17 \mathrm{~mm}$ wide, cool season growth to $1.5 \mathrm{~cm}$ long 'Aurea'

19a. Warm season growth 0.9-2 cm long, 9-14 mm wide; warm season leaves 5-10 mm long; foliage dark green in summer....

'T ansu'

19b. Warm season growth 3.5-7.5 cm long, 12-30 mm wide; warm season leaves 9-17 mm long; foliage medium green in summer

20a. Compact shrub, broad pyramidal to subglobose, to $2.5 \mathrm{~m}$ tall; male strobili complex 1-3, terminal on secondary and tertiary shoots; winter foliage color bronze to purplish-red

20b. D warf shrub forming low spreading mound, to $0.7 \mathrm{~m}$ tall; male strobili complex 2-5 fused strobili, proximal on primary and secondary shoots; winter foliage dark green to bronze

'E legans C ompacta'

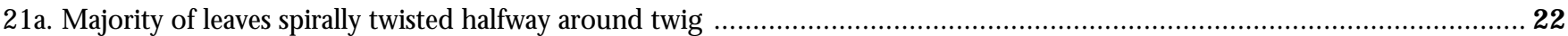

21b. M ajority of leaves lacking spirally twisted leaf shaft

22a. Warm season growth 9-14 mm wide, 2-4 cm long; foliage medium green all year; warm season leaves 10-20 mm long, majority spirally twisted around twig; tree.

'Spiralis'

22b. Warm season growth 4-9 mm wide, 4-7 cm long; foliage light green all year; warm seasonal leaves 9-15 mm long, variable in degree of twisting; shrub

'Spiraliter Falcata'

23a. N ew foliage greenish-yellow or creamy silvery-white.

23b. N ew foliage light to medium green or bluish-green

24a. Warm season growth 2.5-4.2 cm long; cool season growth 0.8-2.7 cm long; warm season leaves slightly incurved, attached at 30-45 angle; new foliage creamy silvery white, becoming light green in summer and bronze in winter

'N ana Albospica'

24b. Warm season growth 1-2.5 cm long; cool season growth 4-12 cm long; warm season leaves slightly recurved, attached at 45$60^{\circ}$ angle; foliage greenish-yellow all year

'L obbii N ana Aurea'

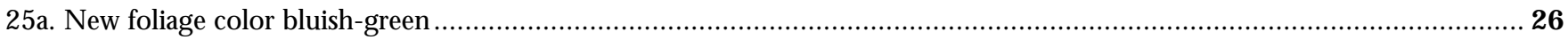

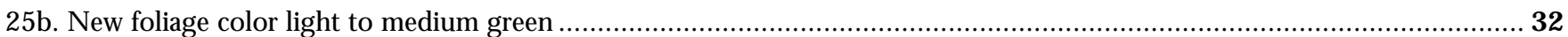

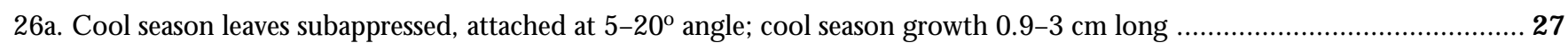

26b. Cool season leaves ascending, attached at $30-85^{\circ}$ angle; cool season growth $0.3-1.6 \mathrm{~cm}$ long .....................................28

27a. Primary shoot 5-6.5 cm long; secondary shoots few, 5-8; warm season growth 0.8-1.5 cm long, 6-7 mm wide; dwarf shrub.

'Little D iamond'

27b. Primary shoot 8.5-19.5 cm long; secondary shoots several, 7-23; warm season growth 1.5-9 cm long, 7-11 mm wide; compact shrub

'Bloomers Witches Broom'

28a. D warf shrub; primary shoots $2.5-5 \mathrm{~cm}$ long; cool season leaves subperpendicular, attached at $75-85^{\circ}$

'Tenzan'

28b. Compact shrub; primary shoots 4-19 cm long; cool season leaves ascending, attached at (5) 30-450

29a. Winter foliage color purplish-red; cool season leaf apex curved; warm season growth elongate, (1) 3-9 cm long; shrubs subglobose.....

29b. Winter foliage color medium to dark green; cool season leaf apex straight; warm season growth short, 1-3.2 cm; shrubs conical to columnar

30a. Primary shoots 11-19 cm long; tertiary shoots present, 3-6, 5-25 mm long; warm season leaves attached at 10-450; leaves slightly incurved; larger secondary shoots to $14 \mathrm{~cm}$

'G lobosa'

30b. Primary shoots 3.5-12 cm long; tertiary shoots lacking; warm season leaves attached at 40-60\%; leaves nearly straight; larger secondary shoots to $7.2 \mathrm{~cm}$ long

'G lobosa N ana' 


\section{Fig. 2. Continued.}

31a. Leaves strongly ascending, attached 30-450; foliage dark green in summer and winter; larger secondary shoots to $4 \mathrm{~cm}$ long; columnar shrub

'B lack D ragon'

31b. Leaves spreading-ascending, attached $45-60^{\circ}$; foliage medium green in summer and winter; larger secondary shoots to $14 \mathrm{~cm}$ long; conical shrub....

'G iokumo'

32a. Cool season leaves spreading, attached $45-80^{\circ}$; number of cool season secondary shoots greater than number of warm seasonal

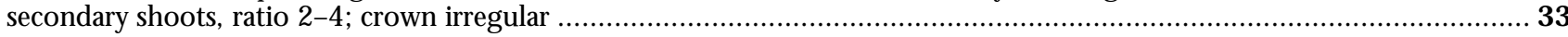

32b. Cool season leaves ascending, attached (5) 30-450; number of cool season secondary shoots nearly equivalent with number of warm season secondary shoots, ratio $0.8-1.2$; crown regular ............................................................................. 36

33a. Warm season growth 1-1.5 cm long; warm season leaves attached at $45-90^{\circ}$; new foliage color light green; tertiary shoots to $2.5 \mathrm{~cm}$ long; quaternary shoots lacking

33b. Warm season growth 2-35 cm long; warm season leaves attached at 20-45; new foliage color medium green; tertiary shoots to $18 \mathrm{~cm}$ long; quaternary shoots sometimes present, to $1 \mathrm{~cm}$ long....

34a. Secondary shoots of warm season growth 4-8, 9-20 in cool season growth; warm season leaves 6-9 mm long; primary shoots 3-5 cm; dwarf shrub

'Yokohama'

34b. Secondary shoots of warm season growth 10-20, 20-80 in cool season growth; warm season leaves 11-19 mm long; primary shoots to $14 \mathrm{~cm}$ long; compact shrub 'Bandai-sui'

35a. Primary shoots 8-42 cm long; warm season growth 4-36 cm long; winter foliage color bronze; small tree 'M onstrosa'

35b. Primary shoots $8-11 \mathrm{~cm}$ long; warm season growth 2-5 cm; winter foliage color medium green; compact shrub

'M onstrosa N ana'

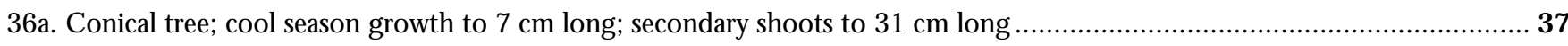

$36 \mathrm{~b}$. Shrub to small tree; cool season growth $0.3-3 \mathrm{~cm}$ long; secondary shoots $0.5-11 \mathrm{~cm}$ long ........................................39

37a. Tertiary shoots $0.4-1.8 \mathrm{~cm}$ long; quaternary shoots to $5 \mathrm{~cm}$ long when present; secondary shoots to $31 \mathrm{~cm}$ long; winter foliage color bronze.

'Yoshino'

37b. Tertiary shoots (0.4) $2-9 \mathrm{~cm}$ long; quaternary shoots lacking or $0.5-0.7 \mathrm{~cm}$ long when present; secondary shoots to $23 \mathrm{~cm}$ long; winter foliage color dark green to brown

38a. Primary shoots $13-29 \mathrm{~cm}$ long; winter foliage color dark green; secondary shoots to $16 \mathrm{~cm}$ long; tertiary shoots to $9 \mathrm{~cm}$ long; quaternary shoots absent; warm season growth $3.5-6 \mathrm{~cm}$ long

38b. Primary shoots 20-55 cm long; winter foliage color brown; secondary shoots to $23 \mathrm{~cm}$ long; tertiary shoots to $6 \mathrm{~cm}$ long; quaternary shoots sometimes present, 5-7 mm long; warm season growth 2-17.5 cm long..... 'Lobbii'

39a. Warm season growth 17-23 mm wide; warm season leaves 8-14 mm long; secondary shoots to $3.5 \mathrm{~cm}$ long......... 'K ukamiga' 39b. Warm season growth 5-17 mm wide; warm season leaves 6-11 mm long; secondary shoots 2.5-8 (11) cm long ...............40 40a. N ew foliage medium green, dark green in summer; tertiary shoots $(0.5)$ 3-6 cm long ........................................... 41 40b. N ew foliage light green, medium green in summer; tertiary shoots $0.3-2.5 \mathrm{~cm}$ long 43

41a. Primary shoots 3.5-8 (12) cm long; tertiary shoots lacking; warm seasonal leaves straight; dwarf shrub 'Vilmoriniana' 41b. Primary shoots (6.5) 9-26 cm long; tertiary shoots present occasionally, 4-20 mm long; compact shrub to small tree 42

42a. Warm season growth $0.8-2 \mathrm{~cm}$ long; cool season growth $0.5-1.5 \mathrm{~cm}$ long; secondary shoots $1.5-8 \mathrm{~cm}$ long; male strobili oblong, 2-10 mm long

'Jindai-sui'

42b. Warm season growth 2-5 cm long; cool season growth to $3 \mathrm{~cm}$ long; secondary shoots 6-11 cm long; male strobili ovoid, 3-6 $\mathrm{mm}$ long

'T aisho-tama'

43a. Warm season growth $1.5-7 \mathrm{~cm}$ long; cool season growth 3-4 $\mathrm{mm}$ wide

'Buckiscope'

43b. Warm season growth $0.8-2 \mathrm{~cm}$ long; cool season growth $4-7 \mathrm{~mm}$ wide 44

44a. Cool season growth $0.4-0.8 \mathrm{~cm}$ long; leaves slightly incurved, apex inflexed; secondary shoots 3-6; dwarf shrub

'Littleworth D warf'

44b. Cool season growth to $2.6 \mathrm{~cm}$ long; leaves straight to recurved, apex straight to reflexed; secondary shoots $6-10$

'G reen Pencil'

a different term for the leaves. In our opinion, the leaves of japanese cedar do not agree with any of these terms previously reported. The leaves of japanese cedar are different in shape and cross-section from awns, needles, awl-shaped, acicular and subulateleaves defined for other genera ( $D$ en $O$ uden and Boom, 1978; Krüssmann, 1985; Tripp, 1993; Welch, 1991, 1993). Adult foliage leaves were quadrangular or compressed dorsoventrally, and similar in appearance to a saber in lateral view. H ence, these leaves are defined herein as saber leaves ( $T$ able 1). L inear leaves were similar to coty- ledon leaves and may represent a juvenile trait. Glaucous bands may be present or lacking on both surfaces of linear leaves.

Femalestrobili are initiated in the summer, develop through the first fall and overwinter. These are receptive to pollination early the following spring. 
Seeds are dispersed in the second fall. $M$ ature strobili may, or may not, persist on the shoot for another (third) year. $M$ ale strobili were more diagnostic for cultivar identification. Cultivars with dwarf or compact growth habits typically lacked female and male strobili, even with age (20-40 years old). Thus, descriptions of cultivars may lack strobili data or be designated as rare when found in a sample size too small to be quantified.

Figure 1 provides an alphabetized checklist of cultivar namesencountered. The 45 recognized cultivars include synonymy, a quantitative morphological description, herbarium vouchers, authorities, and observations. M any selections of japanese cedar were published originally as a distinct speciesor as a variety, but later reduced to the rank of cultivar, the current classification rank. $\checkmark$ ariety and cultivar are not equivalent or synonymous terms, contrary to the understanding and misuse by some in the green industry and the A SH S community (Fantz, 1996). Synonyms are alternative names found in literature or used in horticulture. These names are included in brackets when the cultivar is described, and cultivar names recognized as synonymsareal phabetized and followed by the proper cultivar name that should be used.

Article 17.9 of the International Code of N omenclature for Cultivated Plantsor I CN CP (T rehane, 1995) stated "To be established, a new cultivar epithet published on or after 1 J anuary 1959 must be a word or words in a modern language; Latin wordsor words which may be considered to be Latin, and thus are liable to cause confusion, may not be used unless they are the classical name of an ancient Roman person, or of a place". The Latinized cultivar name is cited in these cases to minimize confusion in the green industry, but a new name agreeing with the Code is proposed.

Five cultivars bear the epithet "elegans" in their name, implying a cultivar group. Four arecompact shrubs to small treeswith spreading linear leaves. H owever, 'Elegans Compacta' has ascending saber leavesand adwarf growth form, thus quite dissimilar. ' $\mathrm{N}$ ana' is closely related to 'E legans Compacta', but often misidentified in American gardens as 'Elegans $\mathrm{N}$ ana'.

Cultivars grown in southern areas exhibited many characters with similar measurement ranges as those grown in northern areas. These characters were regarded as more reliable, thus used in preparing ataxonomic key (Figure2) to assist in segregation and identification of the forty-five cultivars recognized.

\section{Conclusion}

The large number of cultivars selected of japanese cedar provided problemsin segregation and theproper name to use (Rouse et al., 1997). This treatment providesnew quantitativedescriptionsbased upon field data, propernames with synonyms, and a taxonomic key to assist professional plantsmen in segregation and identification of cultivated taxa in the eastern U nited States.

\section{Literature cited}

Beissner, L. 1891. H andbuch der nadelholzkunde. 1st ed. Paul Parley, Berlin.

Beissner, L. 1901. M itteilungen der D eutschen D endrologischen Gesellschaft 10:79.

Beissner, L . and J . Fitschen. 1930. $\mathrm{H}$ andbuch der nadelholzkunde. 3rd ed. Paul Parley, Berlin.

Carrière, E.A. 1867. T raitégénéral desconifères. 2nd ed. Carrière, Paris.

Carrière, E.A. 1876. Rev. H ort. 47:340.

D allimore, W. and A.B. J ackson. 1948. A handbook of Coniferae including Ginkgoaceae. 3rd ed. E. Arnold, London.

Den O uden, P. and B.K. Boom. 1949. Coniferen, ephedraen ginkgo. M artinusN ijhoff, TheH ague, $\mathrm{N}$ etherlands.

Den O uden, P. and B.K. Boom. 1965. M anual of cultivated conifers. $\mathrm{M}$ artinusN ijhoff, TheH ague, $\mathrm{N}$ etherlands.

D en O uden, P. and B.K. Boom. 1978. M anual of cultivated conifers (photomechanical reprint of 1965 ed.). M artinus N ijhoff, The $\mathrm{H}$ ague, $\mathrm{N}$ etherlands.

Dirr, M.A. 1990. M anual of woody landscape plants. 4th ed. Stipes Publ., C hampaign, III.

Eiselt, M.G., 1960. Nadelgeholze. Radebeul N eumann, Berlin.

Fantz, P.R. 1996. N ew cultivar documentation needs to be upgraded. H ortScience 31(5):727728.

Fitschen, J. 1930. In: L. Beissner'shandbuch der nadelholzkunde. 3rd ed. Paul Parley, Berlin.

Grootendorst. 1977. D endroflora 69:13-14.

H arris, J.G. and M.W. H arris. 1994. Plant identification terminology. Spring L ake Publ., Spring Lake, U tah.

H atch, L.C. 1985. Reference guide to ornamental plant cultivars. Taxon. Computer Res., Raleigh, N.C.

$\mathrm{H}$ illier. 1964. The $\mathrm{H}$ illier manual of trees and shrubs. Redwood Press L td., Wiltshire, England.
H ornibrook, M . 1923. D warf and slow-growing conifers. 1st ed. Country Life Ltd., London.

H ornibrook, M. 1924. The Garden 87:37.

H ornibrook, M . 1938. D warf and slow-growing conifers. 2nd ed. Country Life Ltd., London.

Knight, J. and Perry. 1850. A synopsis of the coniferous plants grown in Great Britain. L ongman, Brown, Green, and L ongmans, London.

Kortmann. 1987. D endroflora 24:66.

Krüssmann, G. 1972. M anual of cultivated conifers. 1st ed. Verlag Paul Parey, Berlin.

Krüssmann, G. 1985. M anual of cultivated conifers. 2nd ed. H. Warda (ed.). Timber Press, Portland, Ore.

Laar. 1983. D endroflora 20:83.

Laar. 1990. D endroflora 27:47.

Lyttel. 1934. M y Garden 7:360.

M akoy. 1864. Belgique H ort. 14:356.

Rouse, R.J., P.R. Fantz, and T.E. Bilderback. 1997. Problems identifying japanese cedar cultivated in the U nited States. H ortTechnology 7(2):129-133.

von Billain, B. 1853. In: C.E. Otto and A.G. Dieterich. Allgemeine Gartenzeitung 21:233234.

von Siebold, P.F. 1856. Catalogue raisonné. $\mathrm{H}$ enry $\&$ Cohen, Leiden, $\mathrm{N}$ etherlands.

von Siebold, P.F. 1861. Catalogue Prodromus. $\mathrm{H}$ ollandica, Leiden, $\mathrm{N}$ etherlands.

von Siebold, P.F. and J.G. Zuccarini. 1844. Cryptomeria. Flora japonica 2:42.

Trehane, P. (ed.). 1995. International code of nomenclature for cultivated plants. Q uarterjack Publ., Wimborne, England.

Tripp, K.E. 1993. Sugi: The ancient japanese cedar finds new life in a profusion of outstanding cultivar forms. Amer. N urseryman 178(7):26-39.

Veitch, H.J. 1881. A manual of the Coniferae. James Veitch \& Sons, London.

Vidakovic, M. 1991. Conifers morphology and variation. Graficki Zavod H rvatske, Zagred, Croatia.

Welch, H.J. 1966. D warf conifers, a complete guide. CT Branford Co., N ewton, $M$ ass.

Welch, H.J. 1979. M anual of dwarf conifers. Theophrastus, Little Compton, R.I.

Welch, H .J . 1991. The conifer manual. Kluwer Academic Press, Boston.

Welch, H.J . 1993. The world checklist of conifers. Landsman'sBookshop Ltd., H erefordshire, England.

Wilde, S.A. and G.K. Voigt. 1977. M unsell color charts for plant tissues. 2nd ed. M unsell Color, Kollmorgen Corp., Baltimore, $\mathrm{Md}$.

Yokohoma N ursery. 1923. Yokohomo N ursery catalogue. Yokohoma, Japan. 